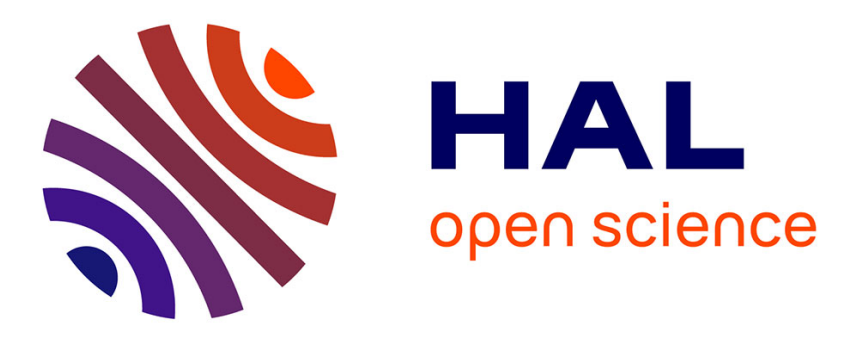

\title{
Sum-Frequency Spectroscopy Amplified by Plasmonics: The Small Particle Case
}

Bertrand Busson, Laetitia Dalstein

\section{To cite this version:}

Bertrand Busson, Laetitia Dalstein. Sum-Frequency Spectroscopy Amplified by Plasmonics: The Small Particle Case. Journal of Physical Chemistry C, 2019, 123, pp.26597-26607. 10.1021/acs.jpcc.9b06334 . hal-02317654

\section{HAL Id: hal-02317654 \\ https://hal.science/hal-02317654}

Submitted on 16 Oct 2019

HAL is a multi-disciplinary open access archive for the deposit and dissemination of scientific research documents, whether they are published or not. The documents may come from teaching and research institutions in France or abroad, or from public or private research centers.
L'archive ouverte pluridisciplinaire HAL, est destinée au dépôt et à la diffusion de documents scientifiques de niveau recherche, publiés ou non, émanant des établissements d'enseignement et de recherche français ou étrangers, des laboratoires publics ou privés. 
$\underline{\text { Sum-Frequency Spectroscopy Amplified by Plasmonics: the Small Particle Case }}$

B. Busson ${ }^{1 *}$, L. Dalstein ${ }^{1,2}$

1) Laboratoire de Chimie Physique, CNRS, Univ. Paris-Sud, Université Paris-Saclay, Bâtiment 201 P2, 91405 Orsay, France

2) Institute of Physics, Academia Sinica, Taipei 11529, Taiwan

Corresponding author: bertrand.busson@u-psud.fr; Tel: +331691532 75; Fax: +331691533 28 


\begin{abstract}
The infrared-visible Sum-Frequency Generation (SFG) spectroscopy response of a composite interface comprising molecules, spherical nanoparticles and a substrate is modelled in the dipolar approximation. The spheres modify the local electric fields felt by the molecules grafted on either the surfaces of the particles or the substrate below the spheres. In the case of plasmonic spheres, the excitation of their surface plasmons lead to amplifications of the molecular SFG signals at the incoming visible and SFG frequencies for both types of molecules. The spectral evolutions of these amplifications are described as a function of the natures of the metal, the molecules and the substrate; the chemical groups involved; the surface density of nanoparticles on the substrate and their surface coverage. The latter parameter is shown to be the main source of SFG from molecules adsorbed on these highly centrosymmetric objects. Models are compared to experimental data and excellent agreement is found for the amplification of the SFG vibrational signature of a grafting monolayer sandwiched between a silicon substrate and gold nanospheres.
\end{abstract}




\section{Introduction}

Sum-Frequency Generation (SFG) spectroscopy is a sensitive, surface or interface-specific experimental technique, widely used as a vibrational spectroscopic tool to analyze molecular chemistry, orientation, adsorption and dynamics at various types of interfaces ${ }^{1}$ and under constraints $^{2,3}$. Its specificity towards interfaces originates in its sensitivity to the symmetries of the probed material: SFG signals are only produced when molecules experience an environment breaking the inversion symmetry. A sensitivity below the monolayer density is therefore attained for highly ordered molecular assemblies at surfaces. On the contrary, when molecules adsorb at an interface in a rather disordered way, SFG sensitivity decreases as a function of molecular disorder. One way to overcome this issue is to compensate for the lack of sensitivity by an experimental amplification of the produced SFG. Several possibilities exist, usually related to a local amplification of the electric fields of light through a coupling to surface plasmon polaritons ${ }^{4}$, resonant microcavities ${ }^{5}$ or evanescent waves in attenuated total reflection geometry ${ }^{6,7}$. Recently, a new route towards amplification has been explored by coupling SFG production to the excitation of localized surface plasmons of nanostructures ${ }^{8-16}$, as happens for SERS for example. Although the use of nanospheres as amplifiers may appear paradoxical as it reintroduces the inversion symmetry for the molecules decorating the spheres, such a scenario remains promising for several reasons: amplification factors are expected to overcome the SFG intensity decrease due to symmetry recovery; spheres are by far not the only possible shapes for plasmonic enhancement; enhancements factors increase in hot spots (i.e. specific locations where the electric fields become giant, for example at the junction between two neighboring nanostructures) where symmetry is broken; inversion symmetry becomes less stringent when the sizes of the particles grow. Finally, we may also mention the possibility to monitor molecules located at the interspace between the nanostructures and the substrate, for which the monolayer symmetries are retained and SFG signals should be enhanced by the sole presence of the particles, as happens in SHINERS experiments ${ }^{17}$. In addition, nanostructured materials made up of particles decorated with molecules and deposited on a substrate are also interesting for themselves, as model sensors or catalysts ${ }^{18,19}$ for example. Developing an experimental tool for the in situ investigation of chemistry happening at their very surfaces helps understanding, complementary to other techniques, their properties and their design.

Extracting chemical information from SFG spectra of an ordered monolayer is a rewarding task, but may already become tricky even in simple cases ${ }^{20,21}$. The presence of nanostructures adds a level of complexity, and modifies the selection rules and analysis procedures developed for monolayers. Understanding how they selectively amplify the various components of the nonlinear signals is therefore a prerequisite for a routine use of such methods.

In order to understand the mechanism of amplification, we develop in a first step the model of SFG production around small isolated supported particles under the dipolar approximation. Such materials do not provide the most intense plasmonic enhancement factors, so much that their amplification is not measurable by SERS as being far too small. However, they provide a good basis for our understanding of the phenomena happening in the close surroundings of the particles, and of the specific effects of local field enhancements of SFG experimental intensities.

\section{Theoretical description of SFG from molecules on a sphere}




\section{A. General considerations}

In the dipole approximation for nanospheres interacting with a light plane wave, the diameter of the spheres $(D=2 R)$ is considered very small as compared to the wavelength of light. Within this approximation, the electric field experienced by the particle is homogeneous over its volume and there are no retardation effects due to the particle size. For bigger particles, retardation may be accounted for partially ${ }^{22}$ or fully through Mie theory applied to nonlinear scattering ${ }^{23}$.

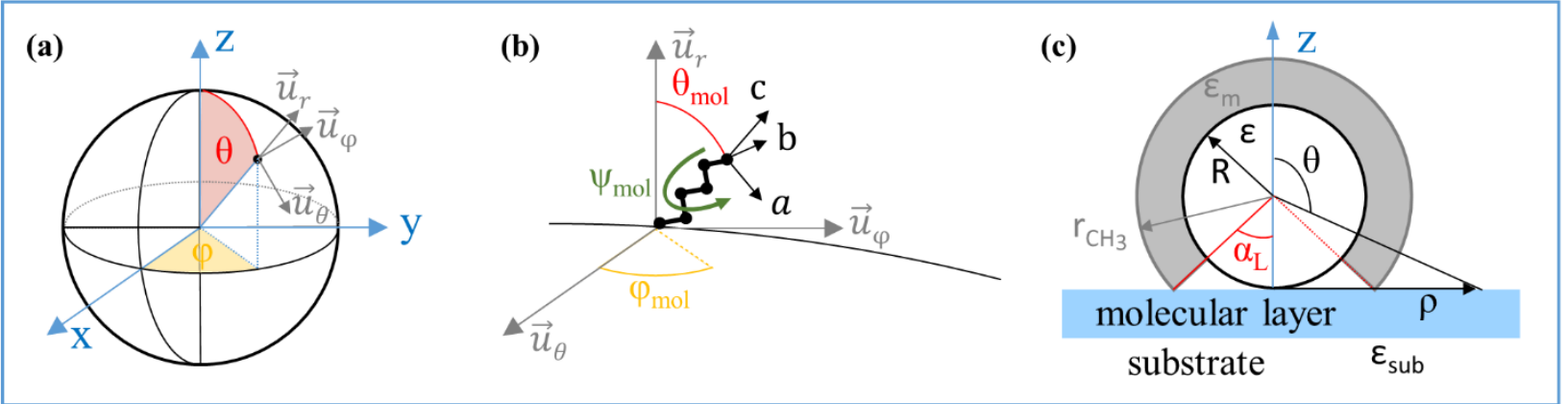

Figure 1 : Definitions of the three frames involved: (a) laboratory $(\boldsymbol{x}, \boldsymbol{y}, \boldsymbol{z})$ and spherical $\left(\boldsymbol{u}_{\theta}, \boldsymbol{u}_{\varphi}, \boldsymbol{u}_{r}\right) ;(b)$ molecular $(\boldsymbol{a}, \boldsymbol{b}, \boldsymbol{c}) ; c)$ Sketch of the samples in the limit angle $\left(\alpha_{L}\right)$ configuration. Molecules in the grey zone are considered in Part II whereas Part III deals with molecules in the blue zone.

We are interested here in isolated and non-interacting nanospheres deposited on a substrate (Figure 1). If we consider molecules decorating the sphere, or molecules in the vicinity of the sphere (for example a monolayer at the surface of the substrate), they will experience a local electric field of light different from the applied far field, and depending on their positions in space.

$$
\begin{aligned}
& \mathbf{E}^{\text {local, }(\mathrm{a}, \mathrm{b}, \mathrm{c})} \stackrel{\mathbf{D}_{\text {mol }}}{\longrightarrow} \mathbf{E}^{\text {local, }(\theta, \varphi, \mathrm{r})} \stackrel{\tilde{\boldsymbol{\Lambda}}}{\longrightarrow} \mathbf{E}^{0,(\theta, \varphi, \mathrm{r})} \stackrel{\mathbf{D}^{0}}{\longrightarrow} \mathbf{E}^{0,(\mathrm{x}, \mathrm{y}, \mathrm{z})} \stackrel{\mathrm{F}_{\mathrm{ik}}}{\longrightarrow} \mathbf{E}^{\mathrm{far},(\mathrm{x}, \mathrm{y}, \mathrm{z})}
\end{aligned}
$$

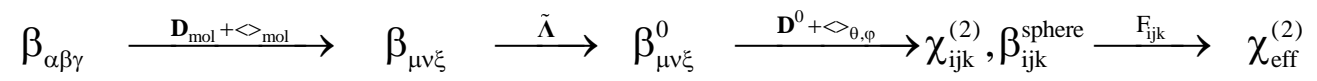

Scheme 1: Definitions and relationships between electric fields, first hyperpolarizabilities and second-order nonlinear susceptibilities involved in the calculation of the SFG response of molecules at the surface of a sphere.

For molecules at the surface of a sphere, the relationships between the molecular local field $\mathbf{E}^{\text {local }}$ and the applied far field $\mathbf{E}^{\mathrm{far}}$ are summarized in Scheme 1. The far field is modified by the reflectivity of the substrate to give rise to the interface field $\mathbf{E}^{0}$. Both are conveniently described by their Cartesian coordinates in the laboratory frame $(\mathbf{x}, \mathbf{y}, \mathbf{z})$ related to $\mathrm{p}$ and s polarizations of light. $\mathbf{E}^{0}$ is then expressed in the spherical coordinates $\left(\mathbf{u}_{\theta}, \mathbf{u}_{\varphi}, \mathbf{u}_{\mathbf{r}}\right)$. The local field effects, which include here plasmonic amplification, are taken into account in the spherical frame through the $\tilde{\Lambda}$ matrix (defined below), to obtain the local field $\mathbf{E}^{\text {local }}$ eventually experienced by the molecule. This field is more conveniently expressed in the molecular frame $(\mathbf{a}, \mathbf{b}, \mathbf{c})$. The actual calculation of all quantities involved in Scheme 1 is performed from the microscopic $\left(\mathbf{E}^{\text {local }}\right)$ to the macroscopic $\left(\mathbf{E}^{\text {far }}\right)$ levels.

In the SFG process, the various fields above relate to several susceptibility and hyperpolarizability tensors. Molecular hyperpolarizability $\beta_{\alpha \beta \gamma}$ (where $\alpha, \beta, \gamma=\mathrm{a}, \mathrm{b}$ or c) relates the local second order dipole moment to the local electric fields through 
$\mathrm{p}_{\alpha}^{\text {local }}\left(\omega_{3}\right)=\beta_{\alpha \beta \gamma} \mathrm{E}_{\beta}^{\text {local }}\left(\omega_{1}\right) \mathrm{E}_{\gamma}^{\text {local }}\left(\omega_{2}\right)$

where $\omega_{1}$ and $\omega_{2}$ stand for the incident visible and infrared frequencies, respectively, and $\omega_{3}=\omega_{1}$ $+\omega_{2}$ for the sum-frequency. The molecular orientation at the surface of the sphere is described by three molecular Euler angles $\theta_{\mathrm{mol}}, \varphi_{\mathrm{mol}}, \psi_{\mathrm{mol}}$ on which the conversion matrix $\mathbf{D}\left(\theta_{\mathrm{mol}}, \varphi_{\mathrm{mol}}, \psi_{\mathrm{mol}}\right)$ between $(\mathbf{a}, \mathbf{b}, \mathbf{c})$ and $\left(\mathbf{u}_{\theta}, \mathbf{u}_{\varphi}, \mathbf{u}_{\mathbf{r}}\right)$ depends. The $\mathbf{D}$ matrix is formally identical to the usual orientation matrix used to convert observables from the molecular frame to the laboratory frame in the case of planar interfaces ${ }^{24,25}$, and recalled in the Appendix I.

The molecules at the surface of the sphere are supposed to all follow identical probability distributions for their orientations with respect to the surface normal to the sphere. Averaging over the molecular Euler angles leads to an average hyperpolarizability $\beta_{\mu v \xi}$ (where $\mu, v, \xi=\theta, \varphi$ or r)

$\beta_{\mu v \xi}=\sum_{\alpha, \beta, \gamma}\left\langle D_{\mu \alpha} D_{v \beta} D_{\xi \gamma}\right\rangle_{\varphi_{\operatorname{mol}}, \psi_{\operatorname{mol}}, \theta_{\mathrm{mol}}} \beta_{\alpha \beta \gamma}$

distributed over the sphere, which acts on the local fields expressed in spherical coordinates. By construction, the only nonvanishing components of $\beta_{\mu \nu \xi}$ are those allowed by the $\mathrm{C}_{\propto \circ v}$ symmetry (respectively $\mathrm{C}_{\infty}$ if the molecule is chiral). The local fields may experience a local enhancement as a consequence of the presence of the sphere and/or the substrate. If we express this enhancement in the spherical coordinates, then

$\mathbf{E}^{\mathrm{local},(\theta, \varphi, \mathrm{r})}\left(\omega_{\mathrm{i}}\right)=\tilde{\boldsymbol{\Lambda}}_{\mathrm{i}}^{(\theta, \varphi, \mathrm{r})} \mathbf{E}^{0,(\theta, \varphi, \mathrm{r})}\left(\omega_{\mathrm{i}}\right)$

Conversely, the relationship between the hyperpolarizabilities expressed in terms of local fields $\left(\beta_{\mu \nu \xi}\right)$ and in terms of interface fields $\left(\beta_{\mu v \xi}^{0}\right)$ is

$\beta_{\mu \nu \xi}^{0}=\sum_{\mu v^{\prime} \xi^{\prime}}\left({ }^{\mathrm{t}} \tilde{\boldsymbol{\Lambda}}_{3}\right)_{\mu \mu^{\prime}}\left({ }^{\mathrm{t}} \tilde{\boldsymbol{\Lambda}}_{1}\right)_{v v^{\prime}}\left({ }^{\mathrm{t}} \tilde{\boldsymbol{\Lambda}}_{2}\right)_{\xi \xi^{\prime}} \beta_{\mu^{\prime} v^{\prime} \xi^{\prime}} \equiv \sum_{\mu^{\prime} v^{\prime} \xi^{\prime}} 1_{\mu^{\prime} v^{\prime} v^{\prime} \xi} \beta_{\mu^{\prime} v^{\prime} \xi^{\prime}}$

At this stage, we may sum up all molecular contributions distributed over the surface of the sphere in order to define the hyperpolarizability of the entire sphere $\left(\beta^{\mathrm{NP}}\right)$, expressed in the laboratory frame. The orientation matrix $\mathbf{D}^{\mathbf{0}}$ from $\left(\mathbf{u}_{\theta}, \mathbf{u}_{\varphi}, \mathbf{u}_{\mathbf{r}}\right)$ to $(\mathbf{x}, \mathbf{y}, \mathbf{z})$ is formally identical to $\mathbf{D}$ with angle $\psi$ set to zero, and summation for a fixed $r$ runs over values of $\theta$ and $\varphi$ covering the actual distribution of molecules. Polarizability components $\beta_{\mu v \xi}^{0}$ are included in the summation as they may depend on $\theta$ and $\varphi$ through the $\tilde{\Lambda}_{\mathrm{i}}$ matrix elements, and we get

$\beta_{\mathrm{ijk}}^{\mathrm{NP}}=\mathrm{n}_{\mathrm{mol} / \mathrm{NP}} \sum_{\mu, v, \xi}\left\langle\mathrm{D}_{\mathrm{i} \mu}^{0} \mathrm{D}_{\mathrm{jv}}^{0} \mathrm{D}_{\mathrm{k} \xi}^{0} \beta_{\mu \nu \xi}^{0}\right\rangle_{\varphi, \theta}$

where $\mathrm{n}_{\text {mol/NP }}$ counts the number of molecules per sphere. In a macroscopic point of view, we have for the local nonlinear susceptibility $\chi_{\mathrm{ijk}}^{(2)}=\mathrm{N}_{\mathrm{S}}^{\mathrm{NP}} \beta_{\mathrm{ijk}}^{\mathrm{NP}}$ where $\mathrm{N}_{\mathrm{s}}^{\mathrm{NP}}$ is the surface density of spheres.

Finally, the experimental SFG intensity measured in an ppp experiment is 
$\mathrm{I}_{\mathrm{p}}\left(\omega_{3}=\omega_{1}+\omega_{2}\right)=\frac{8 \pi^{3}\left(\omega_{3}\right)^{2}}{\mathrm{c}^{3} \cos ^{2} \theta_{\mathrm{SFG}}}\left|\chi_{\mathrm{eff}, \mathrm{ppp}}^{(2)}\right|^{2} \mathrm{I}_{\mathrm{p}}\left(\omega_{1}\right) \mathrm{I}_{\mathrm{p}}\left(\omega_{2}\right)$

where the effective nonlinear susceptibility $\chi_{\mathrm{eff}, \mathrm{ppp}}^{(2)}=\mathrm{F}_{\mathrm{zzz}} \chi_{\mathrm{zzz}}^{(2)}-\mathrm{F}_{\mathrm{xxz}} \chi_{\mathrm{xxz}}^{(2)}+\mathrm{F}_{\mathrm{zxx}} \chi_{\mathrm{zxx}}^{(2)}-\mathrm{F}_{\mathrm{xzx}} \chi_{\mathrm{xzx}}^{(2)}$ and the Fresnel coefficients $F_{i j k}$ relate the incoming and outgoing far fields to the interface fields, taking into account the reflectivity of the substrate ${ }^{26}$.

\section{B. Application to the methyl case}

We take as an example the vibrationally resonant SFG response of methyl moieties at the end of alkane chains decorating a sphere of radius $\mathrm{R}$ at a distance $\mathrm{r}_{\mathrm{CH} 3}$. With a proper definition of the molecular axes (i.e. $\mathbf{c}$ is the $\mathrm{CH}_{3}$ symmetry axis, and $\mathbf{a}$ an axis perpendicular to $\mathbf{c}$ in one of the three planes of symmetry), the $\mathrm{CH}_{3}$ symmetric stretch has local nonvanishing components $\beta_{\text {ccc }}^{\mathrm{CH}_{3}}$ and $\beta_{\mathrm{aac}}^{\mathrm{CH}_{3}}=\beta_{\mathrm{bbc}}^{\mathrm{CH}_{3}}=\mathrm{r}_{\mathrm{a} / \mathrm{c}} \beta_{\mathrm{ccc}}^{\mathrm{CH}_{3}}$, and for the antisymmetric stretch $\beta_{\mathrm{aca}}^{\mathrm{CH}_{3}}=\beta_{\mathrm{caa}}^{\mathrm{CH}_{3}}=\beta_{\mathrm{bcb}}^{\mathrm{CH}_{3}}=\beta_{\mathrm{cbb}}^{\mathrm{CH}_{3}}=\mathrm{r}_{\mathrm{A} / \beta_{\mathrm{ccc}}} \beta_{\mathrm{cos}}^{\mathrm{CH}_{3}} 27,28$.

Formulas for the determination of $\beta_{\mathrm{rrr}}, \beta_{\theta \theta \mathrm{r}}=\beta_{\varphi \varphi \mathrm{r}}$ and $\beta_{\theta \mathrm{r} \theta}=\beta_{\varphi \mathrm{r} \varphi}=\beta_{\mathrm{r} \theta \theta}=\beta_{\mathrm{r} \varphi \varphi}$ coincide with those established in the classical averaging of alkyl chains adsorbed on a plane interface, and may be adapted from the literature ${ }^{28,29}$. All $\varphi_{\mathrm{mol}}, \psi_{\mathrm{mol}}$ orientations are equiprobable, and, supposing that the molecules form a slightly bent SAM at the surface of the sphere (see below the discussion in Part IV), we assume a single tilt angle $\theta_{\mathrm{CH} 3}$ for all molecules as is usually seen on planar surfaces ${ }^{30}$. Generalization to any distribution for the molecular tilt angle is easily implemented ${ }^{31}$ but is not required at this stage.

$$
\begin{aligned}
& \beta_{\mathrm{rrr}}^{\mathrm{SS}}=\beta_{\mathrm{ccc}}^{\mathrm{CH}_{3}}\left[\mathrm{r}_{\mathrm{a} / \mathrm{c}} \cos \theta_{\mathrm{CH}_{3}}+\left(1-\mathrm{r}_{\mathrm{a} / \mathrm{c}}\right) \cos ^{3} \theta_{\mathrm{CH}_{3}}\right] \\
& \beta_{\theta \theta \mathrm{r}}^{\mathrm{SS}}=\beta_{\varphi \varphi \mathrm{r}}^{\mathrm{SS}}=\frac{1}{2} \beta_{\mathrm{ccc}}^{\mathrm{CH}_{3}}\left[\left(1+\mathrm{r}_{\mathrm{a} / \mathrm{c}}\right) \cos \theta_{\mathrm{CH}_{3}}-\left(1-\mathrm{r}_{\mathrm{a} / \mathrm{c}}\right) \cos ^{3} \theta_{\mathrm{CH}_{3}}\right] \\
& \beta_{\theta \mathrm{r} \theta}^{\mathrm{SS}}=\beta_{\varphi \mathrm{r \varphi} \varphi}^{\mathrm{SS}}=\beta_{\mathrm{r} \theta \theta}^{\mathrm{SS}}=\beta_{\mathrm{r} \varphi \varphi}^{\mathrm{SS}}=\frac{1}{2} \beta_{\mathrm{ccc}}^{\mathrm{CH}_{3}}\left(1-\mathrm{r}_{\mathrm{a} / \mathrm{c}}\right)\left(\cos \theta_{\mathrm{CH}_{3}}-\cos ^{3} \theta_{\mathrm{CH}_{3}}\right) \\
& \beta_{\mathrm{rrr}}^{\mathrm{AS}}=2 \beta_{\mathrm{aca}}^{\mathrm{CH}}\left(\cos \theta_{\mathrm{CH}_{3}}-\cos ^{3} \theta_{\mathrm{CH}_{3}}\right) \\
& \beta_{\theta \theta \mathrm{r}}^{\mathrm{AS}}=\beta_{\varphi \varphi \mathrm{r}}^{\mathrm{AS}}=-\beta_{\mathrm{aca}}^{\mathrm{CH}_{3}}\left(\cos \theta_{\mathrm{CH}_{3}}-\cos ^{3} \theta_{\mathrm{CH}_{3}}\right) \\
& \beta_{\theta \mathrm{r} \theta}^{\mathrm{AS}}=\beta_{\varphi \mathrm{rr} \varphi}^{\mathrm{AS}}=\beta_{\mathrm{r} \theta \theta}^{\mathrm{AS}}=\beta_{\mathrm{r} \varphi \varphi}^{\mathrm{AS}}=\beta_{\mathrm{aca}}^{\mathrm{CH}_{3}} \cos ^{3} \theta_{\mathrm{CH}_{3}}
\end{aligned}
$$

The heart of the calculation lies in determining the value of the $\tilde{\boldsymbol{\Lambda}}_{\mathrm{i}}$ matrix in every specific situation. Here below, we briefly develop three cases: molecules on an inert sphere, molecules on a polarizable sphere, first without, then with the influence of the substrate.

\section{Molecules on an inert nanosphere}


We first consider that the sphere has no influence on the optical response of the system, except for the geometrical distribution of molecules. A similar approach was introduced in Ref 14 . We therefore take for its dielectric function $\varepsilon=1$, and neglect the change in the dielectric function around the sphere due to the molecules $\left(\varepsilon_{\mathrm{m}}=1\right)$. Here $\tilde{\boldsymbol{\Lambda}}_{\mathrm{i}}$ is therefore the identity matrix and $\beta_{\mu \nu \xi}^{0}$ components identify to $\beta_{\mu v \xi}$.

When fully covered with molecules, it is easy to check that all second order susceptibility components vanish after summing up over the whole inert sphere (i.e. integrating over isotropic $\theta$ and $\varphi$ in equation 5). This confirms a previous analysis on SFG production at the surface of small particles, showing that the amount of signal is negligible for particles with sizes smaller than one micron, above which it originates in retardation effects ${ }^{32}$. This may be understood as we recover a fully centrosymmetric situation for a completely covered particle. Of course, some deviations from this perfect picture (non-sphericity of the particles, disorder in the molecular layer, terms beyond dipolar approximation) or the inclusion of higher order terms in the SFG sources ${ }^{33}$ may lead to the production of a small amount of SFG photons. Experimentally, if the molecules are adsorbed on the sphere only after their grafting onto the substrate, their surface coverage is not complete and a cone with aperture $2 \alpha$ remains free of molecules (Figure 1c). The value of $\alpha$ is a free parameter, and we may only define a limit value $\alpha_{\mathrm{L}}$ for an ideal molecular coverage as illustrated in Figure 1c, with $\cos \alpha_{\mathrm{L}}=\mathrm{R} / \mathrm{r}$. Centrosymmetry is broken and the hyperpolarizability of the sphere becomes

$$
\begin{aligned}
& \beta_{\mathrm{xxz}}^{\mathrm{NP}}=\beta_{\mathrm{yyz}}^{\mathrm{NP}}=\pi(1+\cos \alpha) \mathrm{R}^{2} \mathrm{~N}_{\mathrm{s}}^{\mathrm{mol}}\left\{\left[\beta_{\theta \theta \mathrm{r}}^{0}+\beta_{\theta \mathrm{r} \theta}^{0}+\beta_{\mathrm{r} \theta \theta}^{0}-\beta_{\mathrm{rrr}}^{0}\right]\left\langle\cos ^{3} \theta\right\rangle+\left[\beta_{\varphi \varphi \mathrm{r}}^{0}-\beta_{\theta \mathrm{r} \theta}^{0}-\beta_{\mathrm{r} \theta \theta}^{0}+\beta_{\mathrm{rrr}}^{0}\right]\langle\cos \theta\rangle\right\} \\
& \beta_{\mathrm{xzx}}^{\mathrm{NP}}=\beta_{\mathrm{yzy}}^{\mathrm{NP}}=\pi(1+\cos \alpha) \mathrm{R}^{2} \mathrm{~N}_{\mathrm{s}}^{\mathrm{mol}}\left\{\left[\beta_{\theta \mathrm{r} \theta}^{0}+\beta_{\theta \theta \mathrm{r}}^{0}+\beta_{\mathrm{r} \theta \theta}^{0}-\beta_{\mathrm{rrr}}^{0}\right]\left\langle\cos ^{3} \theta\right\rangle+\left[\beta_{\varphi \mathrm{r} \varphi}^{0}-\beta_{\theta \theta \mathrm{r}}^{0}-\beta_{\mathrm{r} \theta \theta}^{0}+\beta_{\mathrm{rrrr}}^{0}\right]\langle\cos \theta\rangle\right\} \\
& \beta_{\mathrm{zxx}}^{\mathrm{NP}}=\beta_{\mathrm{zyy}}^{\mathrm{NP}}=\pi(1+\cos \alpha) \mathrm{R}^{2} \mathrm{~N}_{\mathrm{s}}^{\mathrm{mol}}\left\{\left[\beta_{\mathrm{r} \theta \theta}^{0}+\beta_{\theta \mathrm{r} \theta}^{0}+\beta_{\theta \theta \mathrm{r}}^{0}-\beta_{\mathrm{rrr}}^{0}\right]\left\langle\cos ^{3} \theta\right\rangle+\left[\beta_{\mathrm{r} \varphi \varphi}^{0}-\beta_{\theta \mathrm{r} \theta}^{0}-\beta_{\theta \theta \mathrm{r}}^{0}+\beta_{\mathrm{rrr}}^{0}\right]\langle\cos \theta\rangle\right\} \\
& \beta_{\mathrm{zzz}}^{\mathrm{NP}}=2 \pi(1+\cos \alpha) \mathrm{R}^{2} \mathrm{~N}_{\mathrm{s}}^{\mathrm{mol}}\left\{\left[\beta_{\mathrm{rrr}}^{0}-\beta_{\mathrm{r} \theta \theta}^{0}-\beta_{\theta \mathrm{r} \theta}^{0}-\beta_{\theta \theta \mathrm{r}}^{0}\right]\left\langle\cos ^{3} \theta\right\rangle+\left[\beta_{\mathrm{r} \theta \theta}^{0}+\beta_{\theta \mathrm{r} \theta}^{0}+\beta_{\theta \theta \mathrm{r}}^{0}\right]\langle\cos \theta\rangle\right\}
\end{aligned}
$$

where $\mathrm{N}_{\mathrm{S}}^{\mathrm{mol}}$ is the surface density of molecules, which may be deduced from atomic spacing at the surface or determined from electrochemical ${ }^{34,35}$, scattering ${ }^{36}$ or microscopy ${ }^{37}$ measurements on a plane surface of the same material as the sphere, and formula A29 is used for numerical applications.

\section{Molecules on a polarizable nanosphere}

If we consider a more realistic sphere, with $\varepsilon \neq 1$, we have to take into account its polarizability, and the induced dipole moments created by interaction with the three light waves. As the polarizability depends on the surroundings of the sphere, we also take into account the dielectric function created by the molecular layer around the sphere $\left(\varepsilon_{\mathrm{m}}\right)$.

In the dipolar view, which has been shown to provide a very good approximation of the electric field in the immediate surroundings of small particles ${ }^{38}$, the local field inside the molecular layer at a distance $r \geq R$ from the surface of the sphere is now 
$\mathbf{E}^{\text {local }}\left(\omega_{\mathrm{i}}\right)=\left(1-\lambda_{\mathrm{i}}\right) \mathbf{E}^{0}\left(\omega_{\mathrm{i}}\right)+3 \lambda_{\mathrm{i}}\left(\mathbf{E}^{0}\left(\omega_{\mathrm{i}}\right) \cdot \mathbf{u}_{\mathrm{r}}\right) \mathbf{u}_{\mathrm{r}}$

with $\lambda_{\mathrm{i}}=\frac{\varepsilon-\varepsilon_{\mathrm{m}}}{\varepsilon+2 \varepsilon_{\mathrm{m}}}\left(\frac{\mathrm{R}}{\mathrm{r}}\right)^{3}$. Retardation effects due to the finite size of the sphere may be taken into account by adding size-dependent terms to the $\lambda_{\mathrm{i}}$ coefficient ${ }^{22}$, and refined modelling includes a full core-shell dipolar response ${ }^{39}$. The amplification matrix in the $\left(\mathbf{u}_{\theta}, \mathbf{u}_{\varphi}, \mathbf{u}_{\mathrm{r}}\right)$ frame becomes

$$
\tilde{\boldsymbol{\Lambda}}_{\mathrm{i}}^{(\theta, \varphi, \mathrm{r})}=\left(\begin{array}{ccc}
1-\lambda_{\mathrm{i}} & 0 & 0 \\
0 & 1-\lambda_{\mathrm{i}} & 0 \\
0 & 0 & 1+2 \lambda_{\mathrm{i}}
\end{array}\right)
$$

and the relationship between $\beta_{\mu \nu \xi}^{0}$ and $\beta_{\mu v \xi}$ becomes

$\beta_{\mu v \xi}^{0}=\sum_{\mu v^{\prime} \xi^{\prime}}\left({ }^{\mathrm{t}} \tilde{\boldsymbol{\Lambda}}_{3}\right)_{\mu \mu^{\prime}}\left({ }^{\mathrm{t}} \tilde{\boldsymbol{\Lambda}}_{1}\right)_{v v^{\prime}}\left({ }^{\mathrm{t}} \tilde{\boldsymbol{\Lambda}}_{2}\right)_{\xi_{\xi} \xi^{\prime}} \beta_{\mu^{\prime} v^{\prime} \xi^{\prime}}=1_{\mu}\left(\lambda_{3}\right) 1_{v}\left(\lambda_{1}\right) 1_{\xi}\left(\lambda_{2}\right) \beta_{\mu v \xi}$

with $1_{\mu}\left(\lambda_{\mathrm{i}}\right)=\left(1-\lambda_{\mathrm{i}}\right)\left[\delta_{\mu \theta}+\delta_{\mu \varphi}\right]+\left(1+2 \lambda_{\mathrm{i}}\right) \delta_{\mu \mathrm{r}}$

In a seminal paper, Creighton ${ }^{40}$ developed an analogous formalism for SERS. In other words, $\beta^{0}$ components are obtained by multiplying $\beta$ components with the same indices by $\left(1-\lambda_{\mathrm{i}}\right)$ for $\theta$ and $\varphi$ indices, and by $\left(1+2 \lambda_{i}\right)$ for $r$ indices, with the enhancements factors $\lambda_{i}$ calculated for a radius $r \geq R$ taking into account the average distance between the surface and the molecular (e.g. $\left.\mathrm{CH}_{3}\right)$ moieties. Equations 8 still hold in this case, and $\beta^{0}$ follows the $\mathrm{C}_{\infty} / \mathrm{C}_{\infty}$ symmetry rules as above.

\section{E. Influence of the substrate}

When the sphere is polarized close to the substrate as in Figure 1, the polarizability of the substrate (dielectric function $\varepsilon_{\mathrm{sub}}$ ) induces the appearance of an image dipole, which in turn modifies the plasmonic properties of the sphere. The sphere and its image interfere like in a dissymmetric dimer. The description of this dimer usually requires a multipolar development ${ }^{41-45}$, and, for plasmonic material, leads to an increase in the local field, in particular between the sphere and the substrate ${ }^{46}$. For a first approach, it is possible to limit the development to the dipole approximation because we do not consider the far field integrated properties like extinction cross sections but focus on the local field very close to the particles ${ }^{47,48}$. The influence of the substrate on the sphere polarizability depends on their distance ${ }^{48}$ and we focus here on the simple case of a sphere nearly touching the substrate (i.e. in the approximation of a vanishing distance). The polarizability of the sphere becomes anisotropic, and factors $\lambda_{\mathrm{i}}$ now depend on the direction of the electric field, namely

$\lambda_{\mathrm{i}}^{\|}=\frac{1}{3} \frac{\varepsilon-\varepsilon_{\mathrm{m}}}{\varepsilon_{\mathrm{m}}+\mathrm{f}_{\mathrm{i}}^{\|}\left(\varepsilon-\varepsilon_{\mathrm{m}}\right)}\left(\frac{\mathrm{R}}{\mathrm{r}}\right)^{3}$ and $\lambda_{\mathrm{i}}^{\perp}=\frac{1}{3} \frac{\varepsilon-\varepsilon_{\mathrm{m}}}{\varepsilon_{\mathrm{m}}+\mathrm{f}_{\mathrm{i}}^{\perp}\left(\varepsilon-\varepsilon_{\mathrm{m}}\right)}\left(\frac{\mathrm{R}}{\mathrm{r}}\right)^{3}$

where $\|$ and $\perp$ refer to the plane of the substrate (i.e. (x,y) and z, respectively), and

$\mathrm{f}_{\mathrm{i}}^{\|}=\frac{1}{3}\left(1-\frac{1}{8} \frac{\varepsilon_{\text {sub }}-\varepsilon_{\mathrm{m}}}{\varepsilon_{\text {sub }}+\varepsilon_{\mathrm{m}}}\right)$ and $\mathrm{f}_{\mathrm{i}}^{\perp}=\frac{1}{3}\left(1-\frac{1}{4} \frac{\varepsilon_{\text {sub }}-\varepsilon_{\mathrm{m}}}{\varepsilon_{\text {sub }}+\varepsilon_{\mathrm{m}}}\right)$ 
We first consider amplification in the $(\mathbf{x}, \mathbf{y}, \mathbf{z})$ frame, and equation 9 becomes

$\mathbf{E}^{\mathrm{local},(\mathrm{x}, \mathrm{y}, \mathrm{z})}\left(\omega_{\mathrm{i}}\right)=\tilde{\Lambda}_{\mathrm{i}}^{(\mathrm{x}, \mathrm{y}, \mathrm{z})} \mathbf{E}^{0,(\mathrm{x}, \mathrm{y}, \mathrm{z})}\left(\omega_{\mathrm{i}}\right)$

with $\tilde{\boldsymbol{\Lambda}}_{\mathrm{i}}{ }^{\mathrm{x}, \mathrm{y}, \mathrm{z})}=\mathbf{1}-\boldsymbol{\Lambda}_{\mathrm{i}}+3 \mathbf{u}_{\mathrm{r}}{ }^{\mathrm{t}} \mathbf{u}_{\mathrm{r}} \boldsymbol{\Lambda}_{\mathrm{i}}$ and $\boldsymbol{\Lambda}_{\mathrm{i}}=\left(\begin{array}{ccc}\lambda_{\mathrm{i}}^{\|} & 0 & 0 \\ 0 & \lambda_{\mathrm{i}}^{\|} & 0 \\ 0 & 0 & \lambda_{\mathrm{i}}^{\perp}\end{array}\right)$.

In the spherical frame, it follows

$\tilde{\boldsymbol{\Lambda}}_{\mathrm{i}}^{(\theta, \varphi, \mathrm{r})}=\left(\mathbf{D}^{0}\right)^{-1}\left(\mathbf{1}-\boldsymbol{\Lambda}_{\mathrm{i}}+3 \mathbf{u}_{\mathrm{r}}{ }^{\mathrm{t}} \mathbf{u}_{\mathrm{r}} \boldsymbol{\Lambda}_{\mathrm{i}}\right) \mathbf{D}^{0}$

and, explicitly,

$$
\tilde{\Lambda}_{\mathrm{i}}^{(\theta, \varphi, \mathrm{r})}=\left(\begin{array}{ccc}
1-\lambda_{\mathrm{i}}^{\|} \cos ^{2} \theta-\lambda_{\mathrm{i}}^{\perp} \sin ^{2} \theta & 0 & \left(\lambda_{\mathrm{i}}^{\perp}-\lambda_{\mathrm{i}}^{\|}\right) \sin \theta \cos \theta \\
0 & \left(1-\lambda_{\mathrm{i}}^{\|}\right) & 0 \\
2\left(\lambda_{\mathrm{i}}^{\|}-\lambda_{\mathrm{i}}^{\perp}\right) \sin \theta \cos \theta & 0 & 1+2 \lambda_{\mathrm{i}}^{\|} \sin ^{2} \theta+2 \lambda_{\mathrm{i}}^{\perp} \cos ^{2} \theta
\end{array}\right)
$$

As $\beta^{0}$ components now depend on the tilt angle $\theta$, equations 8 must be modified according to equation 5. Depending on molecular chirality, the presence of the substrate breaks the local $\mathrm{C}_{\infty \mathrm{v}} / \mathrm{C}_{\infty}$ symmetry for tensor $\beta^{0}$ and reduces it to $\mathrm{C}_{\mathrm{S}} / \mathrm{C}_{1}$, respectively, with $(\mathbf{x}, \mathbf{z})$ as the mirror plane. The hyperpolarizability of the sphere therefore involves more components of the $\beta^{0}$ tensor and becomes

$$
\begin{aligned}
\beta_{\mathrm{xxz}}^{\mathrm{NP}}=\pi(1+\cos \alpha) \mathrm{R}^{2} \mathrm{~N}_{\mathrm{s}}^{\mathrm{mol}} & {\left[\left\langle\left(\beta_{\mathrm{rrr}}^{0}-\beta_{\theta \mathrm{r} \theta}^{0}-\beta_{\mathrm{r} \theta \theta}^{0}\right) \sin ^{2} \theta \cos \theta\right\rangle+\left\langle\beta_{\theta \theta \mathrm{r}}^{0} \cos ^{3} \theta\right\rangle+\left\langle\beta_{\varphi \varphi \mathrm{r}}^{0} \cos \theta\right\rangle\right.} \\
- & \left.\left\langle\left(\beta_{\theta \theta \theta}^{0}-\beta_{\mathrm{r} \theta \mathrm{r}}^{0}-\beta_{\theta \mathrm{rr}}^{0}\right) \sin ^{2} \theta \cos \theta\right\rangle-\left\langle\beta_{\mathrm{rr} \theta}^{0} \sin ^{3} \theta\right\rangle-\left\langle\beta_{\varphi \varphi \theta}^{0} \sin \theta\right\rangle\right] \\
\beta_{\mathrm{xzx}}^{\mathrm{NP}}=\pi(1+\cos \alpha) \mathrm{R}^{2} \mathrm{~N}_{\mathrm{s}}^{\mathrm{mol}}[ & \left\langle\left(\beta_{\mathrm{rrr}}^{0}-\beta_{\theta \theta \mathrm{r}}^{0}-\beta_{\mathrm{r} \theta \theta}^{0}\right) \sin ^{2} \theta \cos \theta\right\rangle+\left\langle\beta_{\theta \mathrm{r} \theta}^{0} \cos ^{3} \theta\right\rangle+\left\langle\beta_{\varphi \mathrm{r} \varphi}^{0} \cos \theta\right\rangle \\
- & \left.\left\langle\left(\beta_{\theta \theta \theta}^{0}-\beta_{\mathrm{rr} \theta}^{0}-\beta_{\theta \mathrm{rr}}^{0}\right) \cos ^{2} \theta \sin \theta\right\rangle-\left\langle\beta_{\mathrm{r} \theta \mathrm{r}}^{0} \sin ^{3} \theta\right\rangle-\left\langle\beta_{\varphi \theta \varphi}^{0} \sin \theta\right\rangle\right] \\
\beta_{\mathrm{zxx}}^{\mathrm{NP}}=\pi(1+\cos \alpha) \mathrm{R}^{2} \mathrm{~N}_{\mathrm{s}}^{\mathrm{mol}}[ & {\left[\left(\beta_{\mathrm{rrr}}^{0}-\beta_{\theta \theta \mathrm{r}}^{0}-\beta_{\theta \mathrm{rr} \theta}^{0}\right) \sin ^{2} \theta \cos \theta\right\rangle+\left\langle\beta_{\mathrm{r} \theta \theta}^{0} \cos ^{3} \theta\right\rangle+\left\langle\beta_{\mathrm{r} \varphi \varphi}^{0} \cos \theta\right\rangle } \\
- & \left.\left\langle\left(\beta_{\theta \theta \theta}^{0}-\beta_{\mathrm{rr} \theta}^{0}-\beta_{\mathrm{r} \theta \mathrm{r}}^{0}\right) \cos ^{2} \theta \sin \theta\right\rangle-\left\langle\beta_{\theta \mathrm{rr}}^{0} \sin ^{3} \theta\right\rangle-\left\langle\beta_{\theta \varphi \varphi}^{0} \sin \theta\right\rangle\right] \\
\beta_{\mathrm{zzz}}^{\mathrm{NP}} 2 \pi(1+\cos \alpha) \mathrm{R}^{2} \mathrm{~N}_{\mathrm{s}}^{\mathrm{mol}} & {\left[\left\langle\left(\beta_{\mathrm{r} \theta \theta}^{0}+\beta_{\theta \theta \mathrm{r}}^{0}+\beta_{\theta \mathrm{r} \theta}^{0}\right) \sin ^{2} \theta \cos \theta\right\rangle+\left\langle\beta_{\mathrm{rrr}}^{0} \cos ^{3} \theta\right\rangle\right.} \\
& \left.-\left\langle\left(\beta_{\mathrm{rr} \theta}^{0}+\beta_{\mathrm{r} \theta \mathrm{r}}^{0}+\beta_{\theta \mathrm{rr}}^{0}\right) \cos ^{2} \theta \sin \theta\right\rangle-\left\langle\beta_{\theta \theta \theta}^{0} \sin ^{3} \theta\right\rangle\right]
\end{aligned}
$$

It is conceivable to introduce higher order multipolar effects in this approach ${ }^{43}$. We note that all components still vanish when the cone half-aperture $\alpha$ does. 


\section{Extension to molecules in a monolayer below the sphere}

We have calculated so far the SFG response of the molecules adsorbed at the surfaces of the spheres. However, the general theoretical frame exposed above is flexible enough to integrate other applications. We develop the example of an extension to the total internal reflection geometry in Appendix II. Here we focus on another important application to the amplification of the SFG response of an adsorbed molecular monolayer (surface density of molecules in the monolayer $\mathrm{N}_{\mathrm{s}}^{\text {layer }}$ ) sandwiched between the substrate and a layer of nanoparticles deposited on top of it. We compare the SFG signals recorded for such a molecular layer (represented in blue on Figure 1) before and after deposition of nanospheres with surface density $\mathrm{N}_{\mathrm{S}}^{\mathrm{NP}}$. This scheme has been applied for example in SERS and more recently SHINERS and SFG spectroscopies, although the nanostructures usually have a bigger size and/or a more complex structure in such applications 17,49-51. Scheme 2 describes the new situation, for which the coupling of the sphere polarizability to the substrate is important. The dipole approximation applies here for small particles when the substrate is a dielectric. For metallic substrates, higher orders must be considered as the surface plasmons of the particles may couple to the delocalized plasmons of the substrate $\left({ }^{44,45,52}\right)$.

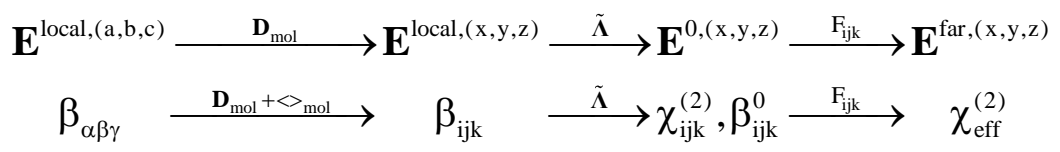

Scheme 2: Definitions and relationships between electric fields, first hyperpolarizabilities and second-order nonlinear susceptibilities involved in the calculation of the SFG response of a molecular layer below a sphere.

The monolayer is supposed known by the molecular $\beta_{\alpha \beta \gamma}$ components and the distribution of molecular Euler angles, giving rise to $\beta_{\mathrm{ijk}}$. Without the enhancement by the spheres, it follows that the average molecular hyperpolarizabilities $\beta_{\mathrm{ijk}}^{0}$ and $\beta_{\mathrm{ijk}}$ coincide, leading to the nonlinear susceptibility expressed as $\chi_{\mathrm{ijk}}^{(2)}=\mathrm{N}_{\mathrm{s}}^{\text {layer }} \beta_{\mathrm{ijk}}^{0}$, and further to the effective nonlinear susceptibility. After deposition of the spheres, the local field is modified by matrix $\tilde{\Lambda}_{\mathrm{i}}$. Using directly

$$
\tilde{\boldsymbol{\Lambda}}_{\mathrm{i}}^{(\mathrm{x}, \mathrm{y}, \mathrm{z})}=\mathbf{1}-\boldsymbol{\Lambda}_{\mathrm{i}}+3 \mathbf{u}_{\mathrm{r}}{ }^{\mathrm{t}} \mathbf{u}_{\mathrm{r}} \boldsymbol{\Lambda}_{\mathrm{i}}
$$

we find

$$
\tilde{\boldsymbol{\Lambda}}_{\mathrm{i}}^{(\mathrm{x}, \mathrm{y}, \mathrm{z})}=\left(\begin{array}{ccc}
1-\lambda_{\mathrm{i}}^{\|}+3 \lambda_{\mathrm{i}}^{\|} \sin ^{2} \theta \cos ^{2} \varphi & 3 \lambda_{\mathrm{i}}^{\|} \sin ^{2} \theta \sin \varphi \cos \varphi & 3 \lambda_{\mathrm{i}}^{\perp} \sin \theta \cos \theta \cos \varphi \\
3 \lambda_{\mathrm{i}}^{\|} \sin ^{2} \theta \sin \varphi \cos \varphi & 1-\lambda_{\mathrm{i}}^{\|}+3 \lambda_{\mathrm{i}}^{\|} \sin ^{2} \theta \sin ^{2} \varphi & 3 \lambda_{\mathrm{i}}^{\perp} \sin \theta \cos \theta \sin \varphi \\
3 \lambda_{\mathrm{i}}^{\|} \sin \theta \cos \theta \cos \varphi & 3 \lambda_{\mathrm{i}}^{\|} \sin \theta \cos \theta \sin \varphi & 1-\lambda_{\mathrm{i}}^{\perp}+3 \lambda_{\mathrm{i}}^{\perp} \cos ^{2} \theta
\end{array}\right)
$$

Every value of $(\theta, \varphi)$ describing the lower half of the sphere points to a molecule in the monolayer which hyperpolarizability components are multiplied by the appropriate matrix coefficients (Figure 1). The surface coverage of the sphere is not as an important parameter as in Part II: we consider here the spheres fully covered with molecules $(\alpha=0)$ because they don't need be functionalized by thiols and are rather deposited with a full coverage of stabilizing agent. The new value for $\beta_{\mathrm{ijk}}^{0}$, under the influence of one sphere, becomes 
$\beta_{\mathrm{ijk}}^{0}=\sum_{\mathrm{i}^{\prime} \mathrm{j}^{\prime} \mathrm{k}}\left({ }^{\mathrm{t}} \tilde{\boldsymbol{\Lambda}}_{3}\right)_{\mathrm{ii}}\left({ }^{\mathrm{t}} \tilde{\boldsymbol{\Lambda}}_{1}\right)_{\mathrm{jj}}\left({ }^{\mathrm{t}} \tilde{\boldsymbol{\Lambda}}_{2}\right)_{\mathrm{kk}} \beta_{\mathrm{i}^{\prime} j^{\prime} \mathrm{j}^{\prime}}$

Due to the form of the $\tilde{\Lambda}_{\mathrm{i}}$ matrix, all nonvanishing $\beta_{\mathrm{i}^{\prime} j^{\prime} \mathrm{k}^{\prime}}$ may contribute to a given $\beta_{\mathrm{ijk}}^{0}$, as long as they survive the averaging step described below. In order to define an amplification factor, we explicitly separate the natural $\left(\beta_{\mathrm{ijk}}\right)$ and amplified $\left(\beta_{\mathrm{ijk}}^{0, \mathrm{NP}}\right)$ contributions to $\beta_{\mathrm{ijk}}^{0}$ :

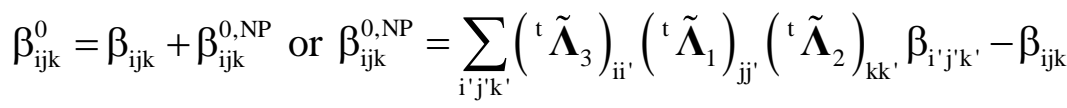

The amplified contribution depends on the position of each molecule with respect to the spheres randomly distributed on the surface, we therefore calculate an average value $\left\langle\beta_{\mathrm{ijk}}^{0}\right\rangle$ of the amplified hyperpolarizability over all molecules in the plane and summed up over all the $\mathrm{n}_{\mathrm{NP}}$ spheres.

$\left\langle\beta_{\mathrm{ijk}}^{0}\right\rangle=\beta_{\mathrm{ijk}}+\left\langle\beta_{\mathrm{ijk}}^{0, \mathrm{NP}}\right\rangle$

We show in the Appendix III that

$$
\left\langle\beta_{\mathrm{ijk}}^{0, \mathrm{NP}}\right\rangle=\mathrm{N}_{\mathrm{S}}^{\mathrm{NP}} \int_{0}^{2 \pi} \int_{\pi}^{\pi / 2} \beta_{\mathrm{ijk}}^{0, \mathrm{NP}} \frac{\mathrm{R}^{2} \sin \theta}{\cos ^{3} \theta} \mathrm{d} \theta \mathrm{d} \varphi
$$

As for the nonlinear susceptibility, we have

$\chi_{\mathrm{ijk}}^{(2)}=\mathrm{N}_{\mathrm{S}}^{\text {layer }}\left\langle\beta_{\mathrm{ijk}}^{0}\right\rangle=\mathrm{N}_{\mathrm{S}}^{\text {layer }} \beta_{\mathrm{ijk}}+\mathrm{N}_{\mathrm{S}}^{\text {layer }}\left\langle\beta_{\mathrm{ijk}}^{0, \mathrm{NP}}\right\rangle$

We may then calculate the amplification factor component by component as

$\mathrm{A}_{\mathrm{ijk}}=\frac{\mathrm{N}_{\mathrm{s}}^{\text {layer }} \beta_{\mathrm{ijk}}+\mathrm{N}_{\mathrm{s}}^{\text {layer }}\left\langle\beta_{\mathrm{ijk}}^{0, \mathrm{NP}}\right\rangle}{\mathrm{N}_{\mathrm{s}}^{\text {layer }} \beta_{\mathrm{ijk}}}=1+\frac{\left\langle\beta_{\mathrm{ijk}}^{0, \mathrm{NP}}\right\rangle}{\beta_{\mathrm{ijk}}}$

or for the overall effective nonlinear susceptibility

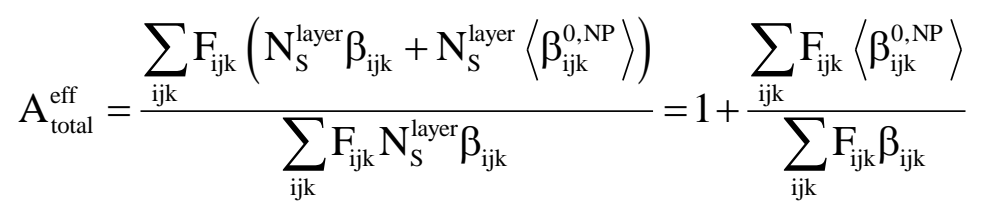

We may illustrate with the example of a monolayer deposited on silicon and used for grafting gold nanoparticles (e.g. APHS or APTES ${ }^{11}$ ). As we will see in more details below, the zzz contribution to the effective nonlinear susceptibility on silicon overcomes all others in the ppp case. We have

$$
\mathrm{A}_{\text {total }}^{\text {eff }} \approx \mathrm{A}_{\text {zzz }}=1+\frac{\left\langle\beta_{\text {zzz }}^{0, \mathrm{NP}}\right\rangle}{\beta_{\text {zzz }}}
$$

with 


$$
\begin{aligned}
\left\langle\beta_{\mathrm{zzz}}^{0, \mathrm{NP}}\right\rangle=2 \pi \mathrm{R}^{2} \mathrm{~N}_{\mathrm{S}}^{\mathrm{NP}} & \left\{\left[\frac{3}{8}\left(\mathrm{G}_{1}^{\perp} \mathrm{G}_{2}^{\perp}+\mathrm{G}_{2}^{\perp} \mathrm{G}_{3}^{\perp}+\mathrm{G}_{3}^{\perp} \mathrm{G}_{1}^{\perp}\right)+\frac{6.8 .10}{7.11 .13} \mathrm{G}_{1}^{\perp} \mathrm{G}_{2}^{\perp} \mathrm{G}_{3}^{\perp}\right] \beta_{\mathrm{zzz}}\right. \\
& +\left[\frac{3}{16} \mathrm{G}_{3}^{\perp} \mathrm{G}_{1}^{\perp}+\frac{14}{11.13} \mathrm{G}_{1}^{\perp} \mathrm{G}_{2}^{\perp} \mathrm{G}_{3}^{\perp}\right]\left(\beta_{\mathrm{xxz}}+\beta_{\mathrm{yyz}}\right) \\
& +\left[\frac{3}{16} \mathrm{G}_{2}^{\perp} \mathrm{G}_{3}^{\perp}+\frac{14}{11.13} \mathrm{G}_{1}^{\perp} \mathrm{G}_{2}^{\perp} \mathrm{G}_{3}^{\perp}\right]\left(\beta_{\mathrm{xzx}}+\beta_{\mathrm{yzy}}\right) \\
& \left.+\left[\frac{3}{16} \mathrm{G}_{1}^{\perp} \mathrm{G}_{2}^{\perp}+\frac{14}{11.13} \mathrm{G}_{1}^{\perp} \mathrm{G}_{2}^{\perp} \mathrm{G}_{3}^{\perp}\right]\left(\beta_{\mathrm{zxx}}+\beta_{\mathrm{zyy}}\right)\right\}
\end{aligned}
$$

where $G_{i}$ factors are defined in Appendix III, and other combinations may be found in the Supporting Information.

IV. Results and discussion: application to real systems

We consider in the following nanospheres (diameter $2 \mathrm{R}=13.5 \mathrm{~nm}$ ) deposited on silicon (refractive index from Ref ${ }^{53}$ ), as we have studied in the past ${ }^{9-12}$, either stabilized by citrate or functionalized by dodecanethiol (DDT) molecules. For dry samples in air, surface charges are balanced and we may neglect third order contributions driven by a local static electric field ${ }^{54-56}$. The differences between gold, silver and silica spheres are investigated for a visible wavelength tunable over a 400$700 \mathrm{~nm}$ range ( $\mathrm{Au}$ and $\left.\mathrm{SiO}_{2}\right)$ or 300-600nm (Ag). Dielectric functions have been tabulated from the literature for gold and silver ${ }^{57,58}$ and, considering the low dispersion for silica in the visible and near-infrared ranges, a single value of 1.5 have been used for its refractive index. For such spheres, the effects of their finite diameters on the dielectric functions may be neglected ${ }^{59}$. As the dielectric function of the DDT monolayer is not precisely known, we have chosen to consider three values for $\varepsilon_{\mathrm{m}}$, analogous to the usual choices for flat surfaces: $\varepsilon_{\mathrm{m}}=\mathrm{n}_{\mathrm{m}}=1$ represents negligible polarizability of adsorbed molecules, an analog of the two layer model for flat surfaces; $\varepsilon_{\mathrm{m}}=1.44$; $\mathrm{n}_{\mathrm{m}}=1.2$ stands for the usual average value of the refractive index of a monolayer in SFG studies 26. $\varepsilon_{\mathrm{m}}=2.1 ; \mathrm{n}_{\mathrm{m}}=1.45$ is a standard bulk value for organic alkane molecules ${ }^{60}$. DDT molecules have a length of $1.77 \mathrm{~nm}$, and the actual distance $\mathrm{r}_{\mathrm{CH} 3}$ of the terminal $\mathrm{CH}_{3}$ moieties to the center of the sphere depends on the molecular tilt angle. The spherical shape is only an approximation for the nanoparticles, and, within this size range, the actual structure is closer to a cuboctahedron ${ }^{61}$. The particles are therefore facetted and it has been shown that thiol molecules adsorb on the facets with a structure similar to self-assembled monolayers on planar gold ${ }^{62,63}$. Even for planar surfaces, there is no agreement in the literature neither on the tilt angle, nor on the relative values of $\beta_{\mathrm{ccc}}, \beta_{\mathrm{aac}}$ and $\beta_{\text {aca. }}$. We have chosen to rely on our previous study of DDT on gold ${ }^{30}$, for which the ratio of $\mathrm{XXz}$ to zzz hyperpolarizability components is consistent with the ratio $\mathrm{r}_{\mathrm{a} / \mathrm{c}}=2.5^{26,28}$ and $\theta_{\mathrm{CH} 3}=33^{\circ}$ ${ }^{64}$. This fixes the distance $\mathrm{r}_{\mathrm{CH} 3}=8.28 \mathrm{~nm}$, and the scale for all $\lambda_{\mathrm{i}}$ to $\left(\mathrm{R} / \mathrm{r}_{\mathrm{CH} 3}\right)^{3}=0.54$. This also gives an estimation of the angle $\alpha_{\mathrm{L}}$, under the hypothesis that the DDT molecules cover the sphere up to the angle for which the $\mathrm{CH}_{3}$ moieties touch the surface of the substrate. In this situation, $\cos \alpha_{\mathrm{L}}=\mathrm{R} / \mathrm{r}_{\mathrm{CH} 3}$ and $\alpha_{\mathrm{L}} \approx 35^{\circ}$. Of course the adsorption geometry of DDT is unknown in practice and the sphere does not exactly touch the substrate, but rather the grafting layer, whose molecular 
structure may be complex ${ }^{65}$. As a consequence, and in order to present a large range of possibilities, we explore several values for $\alpha: 5^{\circ}, 10^{\circ}, 15^{\circ}, 25^{\circ}, 35^{\circ}$ and $45^{\circ}$.

\section{A. Amplified molecular response around the nanoparticles}

We first illustrate the SFG response of DDT molecules surrounding the spheres as calculated in Part II. In the case of the polarizable sphere, three parameters are therefore considered: the nature of the material (gold, silver and silica), the half-aperture of the cone $(\alpha)$ and the dielectric function of the medium surrounding the sphere $\left(\varepsilon_{\mathrm{m}}\right)$. The reference point is $\varepsilon_{\mathrm{m}}=1.44$ and $\alpha=\alpha_{\mathrm{L}}$. A comparison between the dispersions of the amplification parameters $\lambda_{i}$ in the visible range may be found in the Supporting Information (Figure S1). For gold and silver, they show a maximum corresponding to the excitation of the surface plasmon resonances at the visible and SFG frequencies for $i=1$ and 3 , respectively, whereas they remain essentially dispersionless for silica.

Examples of the dispersion of the amplitudes of the local and effective nonlinear susceptibility components for the $\mathrm{CH}_{3}$ symmetric stretch $\left(\beta_{\mathrm{ccc}}=1\right)$ appear in Figure 2 for gold and silver (same curves for silica in the Supporting Information, Figure S2). The presence of both maxima shows that the surface plasmon resonance may couple to the visible and SFG beams. The resonances are narrow and strong for silver, rather broad and less intense for gold as a consequence of interband transition damping. Amplification becomes low for visible wavelengths shorter than the plasmon wavelength, typically below 500nm for gold and 340nm for silver. The maxima redshift and increase with $\varepsilon_{\mathrm{m}}$ as expected. In the case of gold, the redshift couples to a decrease in the excitation of interband transitions, leading to better resolved and higher maxima. As radial components of the electric fields are enhanced by the $\left(1+2 \lambda_{\mathrm{i}}\right)$ factors (as compared to $\left(1-\lambda_{\mathrm{i}}\right)$ for the others), the main contributions stem from $\beta_{\text {rrr }}^{0}$ terms, and the strongest susceptibility components are therefore zzz. The xxz component may sometimes compete with zzz because of the high value of $\beta_{\theta \theta \mathrm{r}}$ and of interference effects between the two peaks. For silver, on the blue side of the spectrum (Figure 2d), the radial amplified and natural contributions to SFG emission essentially cancel each other (i.e. $\left|1+2 \lambda_{3}\right| \sim 0$ ), leading to a very small zzz contribution below $370 \mathrm{~nm}$ and to a damping of the plasmonic resonance with the visible wavelength. For silica (Figure S2 a), the four components compete and their relative weights essentially depend on the molecular hyperpolarizability component ratios. 


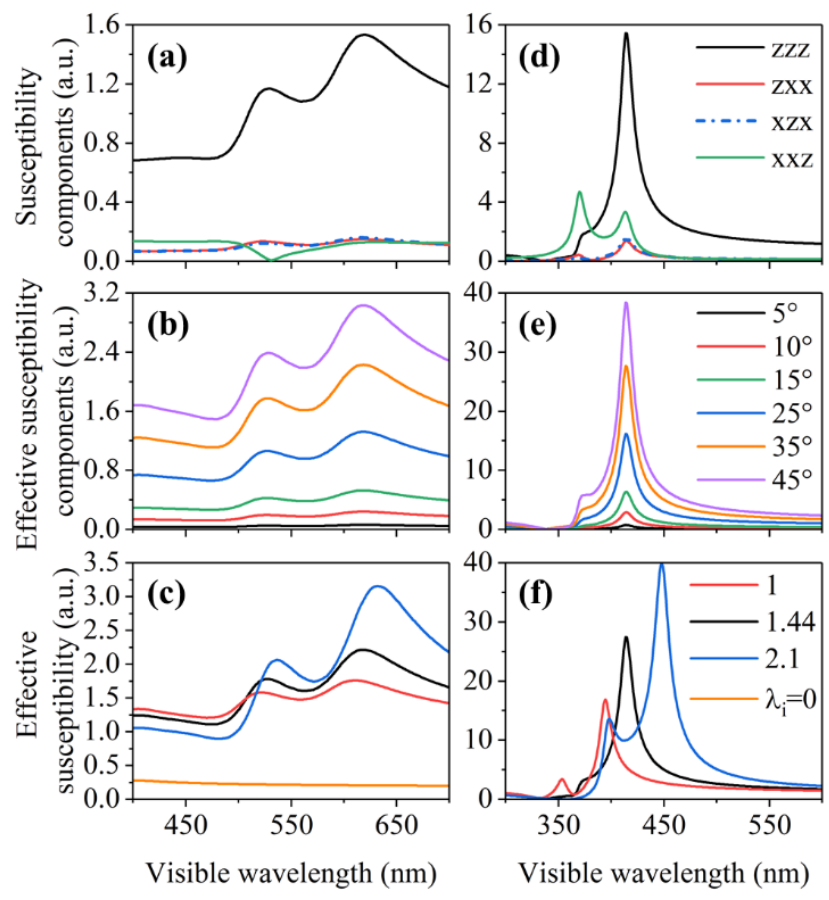

Figure 2: Amplitude variation of nonlinear susceptibilities as a function of incoming visible wavelength for the symmetric stretch of $\mathrm{CH}_{3}$ endgroups of DDT adsorbed on gold $(a, b, c)$ and silver (d,e,f) nanoparticles deposited on silicon, without substrate effects. $(a, d)$ Local $\chi^{(2)}$ components $\left(\varepsilon_{m}=1.44 ; \alpha=35^{\circ}\right) ;(b, e)$ zzz effective $\chi^{(2)}$ component $\left(\varepsilon_{m}\right.$ $=1.44$ ) for six values of angle $\alpha ;(c, f)$ total ppp effective $\chi^{(2)}\left(\alpha=35^{\circ}\right)$ for three values of $\varepsilon_{m}$ and for a void sphere (all $\left.\lambda_{i}=0\right)$.

As for the effective nonlinear susceptibility components, the Fresnel factors account for the low values of the components of the electric fields parallel to the surface as a result of the high reflectivity of silicon. Consequently, at their maxima, the zzz effective components overcome the others by around two orders of magnitude (the situation would substantially differ on a low reflectivity substrate like glass). Considering in equation 8 that, in $\beta_{\mathrm{zzz}}, \beta_{\text {rrr }}^{0}$ is weighted by $<\cos ^{3} \theta>$ and the other components by $\left\langle\cos ^{3} \theta>-<\cos \theta>\right.$, for small values of $\alpha$ the total ppp nonlinear susceptibility is fully dominated by the $\beta^{0}$ rrr term in $\beta_{\text {zzz }}$ (equation A30). As a consequence, the experimental dispersions in the visible range for the symmetric and antisymmetric $\mathrm{CH}_{3}$ stretches, and in general for any vibration mode with a non-vanishing $\beta^{0}$ rrr, shall match and do not depend on the nature of the mode. Only the absolute values will of course differ from mode to mode. Finally, all components increase in amplitude with angle $\alpha$, without any evolution of the lineshapes, because the decrease in the number of molecule at the surface of the spheres does not compensate for the increase in symmetry breaking. A maximum is reached for $\alpha=90^{\circ}$, followed by a symmetric decrease ${ }^{14}$. 


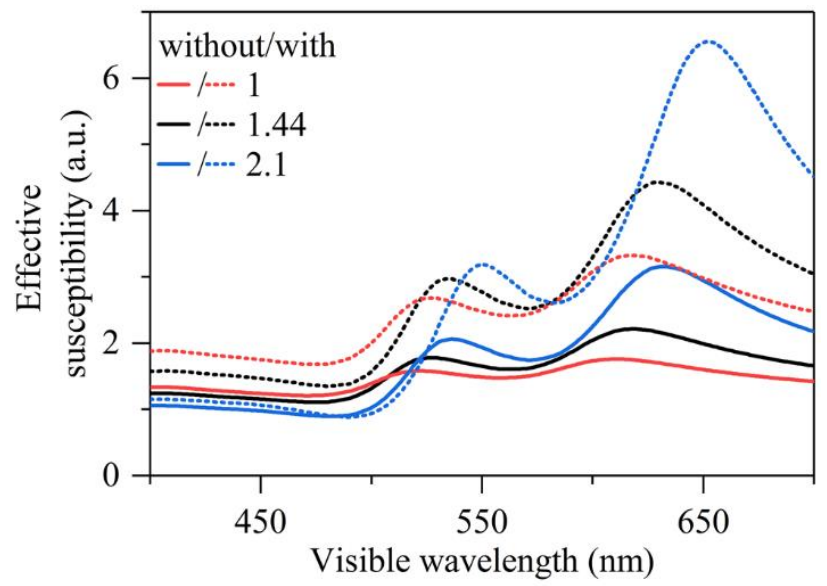

Figure 3: comparison of the variations as a function of incoming visible wavelength of the effective nonlinear susceptibilities for the symmetric stretch of $\mathrm{CH}_{3}$ endgroups of DDT adsorbed on gold nanoparticles deposited on silicon, with and without influence of the substrate for three values of $\varepsilon_{m}\left(\alpha=35^{\circ}\right)$.

When the influence of the substrate is included, the effect on the effective susceptibilities is twofold: a redshift of both maxima and an increase in the amplitude of the response (Figure 3). The redshift may be understood as a consequence of the dipolar interaction between the sphere and its image, as the electric field created by the image always decreases the force acting of the electrons inside the sphere, and consequently their oscillation frequency ${ }^{46}$. The electric field enhancement around the sphere is directly responsible for the increase in amplitude.

\section{B. Amplified molecular response between the particles and the substrate}

We now turn to the case of the grafting monolayer sandwiched between the substrate and the spheres, as calculated in Part III. Contrary to the thiols, we may this time directly compare the SFG intensities produced by the monolayer alone, and by the same monolayer with nanoparticles deposited on top. The particles do not need be functionalized and are usually only covered with their stabilizing agent. On silicon, the zzz effective contribution to SFG will again overwhelm the others as above, either before or after deposition of the particles. The amplitude enhancement attributed to the presence of the nanoparticles therefore directly follows from equations 25 , and more precisely

$$
\mathrm{A}_{\text {total }}^{\text {eff }} \approx 1+2 \pi \mathrm{R}^{2} \mathrm{~N}_{\mathrm{S}}^{\mathrm{NP}}\left[\frac{3}{8}\left(\mathrm{G}_{1}^{\perp} \mathrm{G}_{2}^{\perp}+\mathrm{G}_{2}^{\perp} \mathrm{G}_{3}^{\perp}+\mathrm{G}_{3}^{\perp} \mathrm{G}_{1}^{\perp}\right)+\frac{6.8 .10}{7.11 .13} \mathrm{G}_{1}^{\perp} \mathrm{G}_{2}^{\perp} \mathrm{G}_{3}^{\perp}\right]
$$

For a given choice of metal and substrate, we note that amplification only depends on two parameters, and remains constant whatever the sizes of the spheres, provided that the total surface density covered by the particles remains constant. Such a size-independence is characteristic of the dipolar approximation. The various amplification factors are displayed in the Supporting Information (Figure S3), validating the domination of the $\beta_{\mathrm{zzz}}$ contribution to $\beta_{\mathrm{zzz}}^{0}$ over all other terms, even without Fresnel effects. Figure 4 shows the intensity amplification factor calculated for the three values of $\varepsilon_{\mathrm{m}}$, and with $\mathrm{N}_{\mathrm{S}}^{\mathrm{NP}}=11.610^{10}$ particles per $\mathrm{cm}^{2}$. Depending on the visible wavelength, the SFG signals of the monolayer may be enhanced or decreased upon adsorption of 
the nanoparticles as a consequence of interference between gold and silicon ${ }^{66}$. As for $\varepsilon_{\mathrm{m}}$, we may consider the stabilizing agent as negligible $\left(\varepsilon_{\mathrm{m}}=1\right)$ all the more since it will probably be removed below the spheres upon adsorption, or rely on previous SFG studies on monolayers, which have shown that the actual local refractive index of an organic monolayer is around $n_{m}=1.2^{26,30}$. The amplification values at a visible wavelength of $532 \mathrm{~nm}$ are then $6.3\left(\varepsilon_{\mathrm{m}}=1\right)$ and $6.0\left(\varepsilon_{\mathrm{m}}=1.44\right)$.
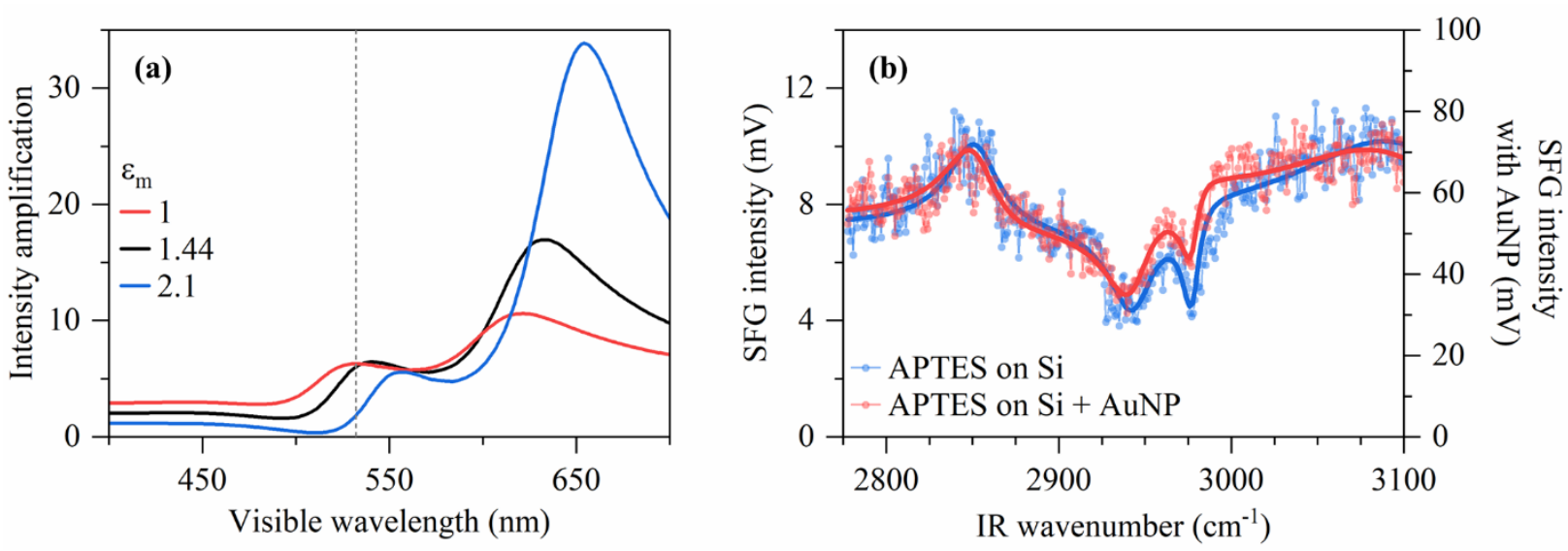

Figure 4: (a) Intensity amplification factors for the vibrational modes in the underlying monolayer for various values of $\varepsilon_{m}$. (b) SFG spectra (visible wavelength: $532 \mathrm{~nm}$ ) in the CH stretch range of an APTES monolayer on silicon, before (blue, left) and after (red, right) grafting of gold nanoparticles. Circles are the experimental points, continuous line a fit to a Lorentzian model. Adapted from Ref. 12. Copyright 2015 American Chemical Society.

These values may be directly compared to experimental measurements. We have deposited a layer of APTES (aminopropyl triethoxysilane) on a silicon wafer after proper cleaning, in the same conditions as in our previous publication ${ }^{12}$. As is now established, APTES condensation on silicon is far from perfect and results in a rather disorganized layer, in which part of the ethoxy methyls are still present ${ }^{65}$. The characteristic methyl vibration modes may be evidenced by SFG before and after nanoparticle deposition of citrate-covered particles (diameter $13.5 \mathrm{~nm}$ ), prepared as explained before $^{12}$. In Figure 4, we show a superimposition of the SFG spectra in the methyl stretch frequency range before and after particle grafting. The surface density of particles was measured as $\mathrm{N}_{\mathrm{S}}^{\mathrm{NP}}=$ $11.610^{10}$ particles per $\mathrm{cm}^{2}$ on several electron microscopy images, which also showed a low level of aggregation. This was confirmed by visible reflectance spectroscopy, showing negligible contribution from interparticle coupling. The experimental spectra show that both the resonant $\left(\mathrm{CH}_{3}\right.$ vibrations) and the nonresonant SFG intensities are enhanced together by a comparable amount as a consequence of nanoparticle deposition. This is not surprising as the grafting layer and the silicon surface, which act as the sources of both signals, are located in the gap between the particles and their images, thus experimenting analogous enhancement factors. From the Figure, the experimental amplification is estimated to 7.1, although it may slightly vary depending on the vibrational features considered. In more details, a fit according to a classical Lorentzian model ${ }^{20,26}$ with four resonances interfering with a nonresonant background gives amplification factors of 7.3, 5.8, 3.1, 6.2 and 5.3 for the NR and the four vibration modes, respectively (see SI for details). 
Amplifications all lie in the same range, which is not surprising considering the selection rules discussed above (Equation 26). We may expect discrepancies between calculated and measured experimental amplification factors on account of the various hypotheses of our model: dipolar and spherical approximations, low interparticle coupling, vanishing distance between the spheres and the underlayer. Comparison between theoretical predictions and experiment shows that the present modelling allows to recover the order of magnitude of the experimental amplification, and validates in particular the use of the dipolar approximation for such systems. Effectiveness of amplification also depends on the disturbance of the monolayer by the grafting of the particles, which may act as an enhancer or reducer of local order. The reproducibility of both the nonresonant and resonant features before and after grafting is a proof that this effect was limited here.

\section{Conclusion}

The dipolar approximation has been used as the first step of a comprehensive modelling of the SFG response of molecules in the surroundings of supported metal nanoparticles, aiming at understanding the experimental amplifications of SFG by plasmonics. Although it does not always provide quantitatively accurate predictions, this approximation allows easy implementation and flexibility, and leads to semi-quantitative results. In the specific case described here, namely particles in the 10-20nm diameter range, the dipolar approach gets close to more sophisticated theories and is sufficient to understand and quantify the observed phenomena and amplification processes.

In this approximation, the useful formulas for the calculation of hyperpolarizability and nonlinear SFG susceptibility components are provided for both functionalization molecules adsorbed at the surface of the particles and a grafting monolayer below the particles. The local amplifications of the electric fields due to the presence of nanospheres are taken into account, as well as the image dipoles created into the substrate. Enhancements by spheres made of silver, gold and silica are compared as a function of the visible wavelength involved in the SFG process, showing as expected that, at resonance with the surface plasmons, silver leads to much higher plasmonic enhancements than gold, whereas no resonant effect is seen with silica. The enhancements depend on the chemical group under scrutiny through their distances to the surface of the sphere, their local order and their balance between molecular susceptibility components. They also vary with the specific properties of the studied interfaces, namely the nature of the substrate, refractive index of the surrounding molecules, surface density of spheres and molecular surface coverage of the nanoparticles.

A direct application is provided by calculating the amplification factor of the SFG spectrum of the APTES grafting layer of gold nanoparticles on silicon and comparing it to the experimental data. We plan to study the response of the thiol layer in a forthcoming dedicated paper. The excellent agreement shows that the methodology provided here is applicable indeed to real systems. More sophisticated descriptions (e.g. mapping the electric field amplitudes calculated by numerical methods in the particle-substrate system) may provide more accurate results and hopefully will complement the present description but they imply more complex theoretical developments and calculations. We believe that, considering the imperfect knowledge on the experimental interfaces 
and their degree of heterogeneity, the models presented here shall remain sufficient for most of the cases.

\section{Supporting information}

Amplification parameters calculated for molecules around gold, silver and silica spheres; hyperpolarizability components for DDT molecules around silica spheres; amplification factors for the molecular monolayer between gold nanoparticles and the silicon substrate; fit parameters for the amplified underlayer system.

\section{Acknowledgement}

We thank Prof. Souhir Boujday and Dr Maroua Ben Haddada (Laboratoire de Réactivité des Surfaces, Paris) for their help in the sample preparation. Research leading to these results was supported by the Région Ile-de-France in the framework of the funding program C'Nano IdF under grant agreement CREMOSOFT. L.D. acknowledges funding from Ministère de l'Enseignement Supérieur.

\section{Appendix I: molecular hyperpolarizabilities around the nanospheres}

We recall the value of the $\mathbf{D}$ matrix defined from the Euler angles

$$
\mathbf{D}=\left(\begin{array}{ccc}
\cos \varphi \cos \theta \cos \psi-\sin \varphi \sin \psi & -\cos \varphi \cos \theta \sin \psi-\sin \varphi \cos \psi & \cos \varphi \sin \theta \\
\sin \varphi \cos \theta \cos \psi+\cos \varphi \sin \psi & -\sin \varphi \cos \theta \sin \psi+\cos \varphi \cos \psi & \sin \varphi \sin \theta \\
-\sin \theta \cos \psi & \sin \theta \sin \psi & \cos \theta
\end{array}\right)
$$

Averaging follows from the integrals

$$
\langle\mathrm{f}\rangle_{\varphi, \psi, \theta}=\frac{1}{8 \pi^{2}} \int_{0}^{2 \pi} \int_{0}^{2 \pi} \int_{0}^{\pi-\alpha} \mathrm{f}(\varphi, \psi, \theta) \sin \theta \mathrm{d} \varphi \mathrm{d} \psi \mathrm{d} \theta
$$

and leads to Eq. 7, 8 and 17. For example,

$$
\left\langle\cos ^{\mathrm{p}} \theta\right\rangle=\frac{1}{2} \int_{0}^{\pi-\alpha} \cos ^{\mathrm{p}} \theta \sin \theta \mathrm{d} \theta=\frac{1}{2(\mathrm{p}+1)}\left(1+(-1)^{\mathrm{p}} \cos ^{\mathrm{p}+1} \alpha\right)
$$

It follows

$$
\frac{\left\langle\cos ^{3} \theta\right\rangle}{\langle\cos \theta\rangle-\left\langle\cos ^{3} \theta\right\rangle}=\frac{1+\cos ^{2} \alpha}{\sin ^{2} \alpha}
$$

For $\alpha=0$, integrals of the form 
$\left\langle\sin ^{\mathrm{q}} \theta \cos ^{\mathrm{p}} \theta\right\rangle=\frac{1}{2} \int_{0}^{\pi} \sin ^{\mathrm{q}} \theta \cos ^{\mathrm{p}} \theta \sin \theta \mathrm{d} \theta$

vanish, whatever the value of $q$, if, and only if, $p$ is odd.

\section{Appendix II: Extension to total internal reflection}

As a second example, we investigate the influence of the total internal reflection geometry (TIR) on the SFG response of nanoparticles deposited on a substrate (e.g. a prism with refractive index $\mathrm{n}_{\mathrm{i}}$, considered as the incoming medium). It is well-known that TIR favors amplification of the SFG molecular signals ${ }^{6,7}$, mainly as a consequence of Fresnel reflectivity enhancements close to the critical angles. When the sample involves molecules decorating nanoparticles, another effect appears which may lead to an amplification of the signal as a consequence of a new symmetry breaking ${ }^{16}$. For a nanosphere fully covered with molecules, for which we have seen that the SFG signal vanishes in conventional reflection geometry, we now have to take into account the fact that the $\mathrm{z}$ component of electric field is evanescent, i.e. it does not propagate in the second medium but rather decreases exponentially as a function of the depth. This creates an up-down asymmetry in the $\mathrm{z}$ direction, giving rise to new contributions to second-order nonlinear processes. It is possible to include this effect in the dipolar regime (which does not take into account other higher order contributions related to the electric field gradients ${ }^{67}$ ). Considering the propagation of a light wave in the second medium where the nanoparticles stand, taken here as air with $n_{\text {air }}=1$, its amplitude decreases as $\mathrm{e}^{-\delta_{\mathrm{i}} \mathrm{z}}$ where $\delta_{\mathrm{i}}=\frac{2 \pi}{\lambda} \sqrt{\mathrm{n}_{\mathrm{i}}^{2} \sin ^{2} \theta_{\mathrm{i}}-1}$ and $\mathrm{z}=\mathrm{R}+\mathrm{r} \cos \theta$ for a sphere touching the surface, or $\mathrm{z}=\mathrm{r}(1+\cos \theta)$ when the sphere is fully decorated with molecules. We therefore modify the $\tilde{\boldsymbol{\Lambda}}_{\mathrm{i}}$ matrix as

$\tilde{\boldsymbol{\Lambda}}_{\mathrm{i}}^{(\theta, \varphi, \mathrm{r}), T \mathrm{TR}}=\mathrm{e}^{-\delta_{\mathrm{i}}(\mathrm{R}+\mathrm{r} \cos \theta)} \tilde{\boldsymbol{\Lambda}}_{\mathrm{i}}^{(\theta, \varphi, \mathrm{r})}$

In the dipolar frame, $\mathrm{z}$ remains small as compared to the wavelength, hence $\mathrm{z} \delta_{\mathrm{i}} \ll<1$. By linearization, we get

$$
\tilde{\boldsymbol{\Lambda}}_{\mathrm{i}}^{(\theta, \varphi, \mathrm{r}), \mathrm{TIR}}=\tilde{\boldsymbol{\Lambda}}_{\mathrm{i}}^{(\theta, \varphi, \mathrm{r})}\left(1-\delta_{\mathrm{i}} \mathrm{R}\right)-\delta_{\mathrm{i}} \mathrm{r} \cos \theta\left(\begin{array}{ccc}
1-\lambda_{\mathrm{i}}^{\|} \cos ^{2} \theta-\lambda_{\mathrm{i}}^{\perp} \sin ^{2} \theta & 0 & \left(\lambda_{\mathrm{i}}^{\perp}-\lambda_{\mathrm{i}}^{\|}\right) \sin \theta \cos \theta \\
0 & \left(1-\lambda_{\mathrm{i}}^{\|}\right) & 0 \\
2\left(\lambda_{\mathrm{i}}^{\|}-\lambda_{\mathrm{i}}^{\perp}\right) \sin \theta \cos \theta & 0 & 1+2 \lambda_{\mathrm{i}}^{\|} \sin ^{2} \theta+2 \lambda_{\mathrm{i}}^{\perp} \cos ^{2} \theta
\end{array}\right)
$$

The consequences are twofold: firstly, when the sphere is not fully covered with molecules (i.e. angle $\alpha$ differs from zero), the first term dominates and the molecular response is calculated by plugging equation (10) into equations (7) and (6). The asymmetry induced by the TIR geometry only marginally influences the overall response and the TIR amplification therefore mainly relies on Fresnel effects. However, when the sphere is fully covered with molecules, the first term vanishes after averaging over the sphere, and asymmetry related to the exponential decrease 
becomes the only source for SFG at this level of approximation. The odd powers of cosine terms ensure that they don't vanish after averaging, however their amplitudes remain low, as the symmetry breaking, quantified by $\delta_{\mathrm{i}}$, is limited. For bigger particles ${ }^{32}$, the effect increases, but the present theory becomes less valid.

\section{Appendix III: amplified hyperpolarizabilities below the nanospheres}

We start by considering how the presence of one sphere modifies the average hyperpolarizability for a molecule in the underlayer and define the average molecular polarizability of the molecules in the monolayer influenced by the presence of one sphere $\left\langle\beta_{\mathrm{ijk}}^{0, \mathrm{NP}}\right\rangle_{1 \mathrm{NP}}$. As all molecules in the plane are influenced by the sphere, $\left\langle\beta_{\mathrm{ijk}}^{0, \mathrm{NP}}\right\rangle_{1 \mathrm{NP}}$ is obtained by an average over the whole plane of the nonlinear response amplified by the local field factors. Seen from the sphere, each molecule experiences an amplified local field which amplitude depends on the variable distance $r$ to the center of the sphere (through the $\lambda_{i}^{\perp, \|}$ factors) and on the angles $\varphi$ and $\theta$ (through the $\tilde{\Lambda}_{\mathrm{i}}$ components).

The dependencies in $\mathrm{r}$ and $\theta$ being related, we define

$\lambda_{\mathrm{i}}^{\perp, \|}=\frac{1}{3} \frac{\varepsilon-\varepsilon_{\mathrm{m}}}{\varepsilon_{\mathrm{m}}+\mathrm{f}_{\mathrm{i}}^{\perp, \|}\left(\varepsilon-\varepsilon_{\mathrm{m}}\right)}\left(\frac{\mathrm{R}}{\mathrm{r}}\right)^{3}=-\frac{1}{3} \frac{\varepsilon-\varepsilon_{\mathrm{m}}}{\varepsilon_{\mathrm{m}}+\mathrm{f}_{\mathrm{i}}^{\perp, \|}\left(\varepsilon-\varepsilon_{\mathrm{m}}\right)} \cos ^{3} \theta=-\mathrm{G}_{\mathrm{i}}^{\perp, \|} \cos ^{3} \theta$

Averaging over $\varphi$ and $\theta$ covers the whole plane and extents between 0 and $2 \pi$, and $\pi / 2$ and $\pi$, respectively. Finally, we may sum up over all spheres $\left(\mathrm{n}_{\mathrm{NP}}\right)$ to calculate the average molecular hyperpolarizability modified by the presence of all the spheres.

$$
\begin{aligned}
& \left\langle\beta_{\mathrm{ijk}}^{0}\right\rangle=\beta_{\mathrm{ijk}}+\left\langle\beta_{\mathrm{ijk}}^{0, \mathrm{NP}}\right\rangle \text { with }\left\langle\beta_{\mathrm{ijk}}^{0, \mathrm{NP}}\right\rangle=\mathrm{n}_{\mathrm{NP}}\left\langle\beta_{\mathrm{ijk}}^{0, \mathrm{NP}}\right\rangle_{1 \mathrm{NP}} \\
& \left\langle\beta_{\mathrm{ijk}}^{0, \mathrm{NP}}\right\rangle=\frac{\mathrm{n}_{\mathrm{NP}}}{\mathrm{n}_{\text {layer }}} \int_{\theta, \varphi} \beta_{\mathrm{ijk}}^{0, \mathrm{NP}} \mathrm{dn} \mathrm{n}_{\text {layer }}=\frac{\mathrm{n}_{\mathrm{NP}} \mathrm{N}_{\mathrm{S}}^{\mathrm{layer}}}{\mathrm{n}_{\text {layer }}} \int_{\theta, \varphi} \beta_{\mathrm{ijk}}^{0, \mathrm{NP}} \mathrm{dS}=\mathrm{N}_{\mathrm{S}}^{\mathrm{NP}} \int_{0}^{\infty} \int_{0}^{2 \pi} \beta_{\mathrm{ijk}}^{0, \mathrm{NP}} \rho \mathrm{d} \rho \mathrm{d} \varphi
\end{aligned}
$$

Using $\rho=-R \tan (\theta)$, we have

$$
\left\langle\beta_{\mathrm{ijk}}^{0, \mathrm{NP}}\right\rangle=\mathrm{N}_{\mathrm{S}}^{\mathrm{NP}} \int_{0}^{2 \pi} \int_{\pi}^{\pi / 2} \beta_{\mathrm{ijk}}^{0, \mathrm{NP}} \frac{\mathrm{R}^{2} \sin \theta}{\cos ^{3} \theta} \mathrm{d} \theta \mathrm{d} \varphi
$$

For the calculation of (A37) using Eq. (18), we take advantage of the following integrals

$$
\int_{\pi}^{\pi / 2} \cos ^{\mathrm{p}} \theta \frac{\sin \theta}{\cos ^{3} \theta} \mathrm{d} \theta=\frac{(-1)^{\mathrm{p}}}{\mathrm{p}-2}
$$

and 


$$
\int_{\pi}^{\pi / 2} \sin ^{2} \theta \cos ^{\mathrm{p}} \theta \frac{\sin \theta}{\cos ^{3} \theta} \mathrm{d} \theta=\frac{2(-1)^{\mathrm{p}}}{\mathrm{p}(\mathrm{p}-2)}
$$

\section{$\underline{\text { References }}$}

(1) Arnolds, H.; Bonn, M. Ultrafast Surface Vibrational Dynamics. Surf. Sci. Rep. 2010, 65, 45-66.

(2) Rupprechter, G. A Surface Science Approach to Ambient Pressure Catalytic Reactions. Catal. Today 2007, 126, 3-17.

(3) Behrens, R. L.; Lagutchev, A.; Dlott, D. D.; Wieckowski, A. Broad-Band Sum Frequency Generation Study of Formic Acid Chemisorption on a Pt (1 000$)$ Electrode. J. Electroanal. Chem. 2010, 649, 32-36.

(4) van der Ham, E. W. M.; Vrehen, Q. H. F.; Eliel, E. R.; Yakovlev, V. A.; Alieva, E. V.; Kuzik, L. A.; Petrov, J. E.; Sychugov, V. A.; van der Meer, A. F. G. Giant Enhancement of Sum-Frequency Yield by Surface-Plasmon Excitation. J. Opt. Soc. Am. B 1999, 16, 11461152.

(5) Mattei, G.; Valentini, V.; Yakovlev, V. A.; Mani, A. A.; Sartenaer, Y.; Thiry, P. A.; Peremans, A.; Caudano, Y.; Dreesen, L.; Humbert, C.; et al. Sum-Frequency Generation from Surface Species in Porous Silicon. Phys. Status Solidi 2005, 202, 1487-1491.

(6) Liljeblad, J. F. D.; Tyrode, E. Vibrational Sum Frequency Spectroscopy Studies at Solid/Liquid Interfaces: Influence of the Experimental Geometry in the Spectral Shape and Enhancement. J. Phys. Chem. C 2012, 116, 22893-22903.

(7) Löbau, J.; Wolfrum, K. Sum-Frequency Spectroscopy in Total Internal Reflection Geometry: Signal Enhancement and Access to Molecular Properties. J. Opt. Soc. Am. B 1997, 14, 2505-2512.

(8) Humbert, C.; Busson, B.; Abid, J. P.; Six, C.; Girault, H. H.; Tadjeddine, A. SelfAssembled Organic Monolayers on Gold Nanoparticles: A Study by Sum-Frequency Generation Combined with UV-Vis Spectroscopy. Electrochim. Acta 2005, 50, 31013110 .

(9) Humbert, C.; Pluchery, O.; Lacaze, E.; Tadjeddine, A.; Busson, B. Optical Spectroscopy of Functionalized Gold Nanoparticles Assemblies as a Function of the Surface Coverage. Gold Bull. 2013, 46, 299-309.

(10) Pluchery, O.; Humbert, C.; Valamanesh, M.; Lacaze, E.; Busson, B. Enhanced Detection of Thiophenol Adsorbed on Gold Nanoparticles by SFG and DFG Nonlinear Optical Spectroscopy. Phys. Chem. Chem. Phys. 2009, 11, 7729-7737.

(11) Humbert, C.; Pluchery, O.; Lacaze, E.; Tadjeddine, A.; Busson, B. A Multiscale Description of Molecular Adsorption on Gold Nanoparticles by Nonlinear Optical Spectroscopy. Phys. Chem. Chem. Phys. 2012, 14, 280-289. 
(12) Dalstein, L.; Ben Haddada, M.; Barbillon, G.; Humbert, C.; Tadjeddine, A.; Boujday, S.; Busson, B. Revealing the Interplay between Adsorbed Molecular Layers and Gold Nanoparticles by Linear and Nonlinear Optical Properties. J. Phys. Chem. C 2015, 119, 17146-17155.

(13) Weeraman, C.; Yatawara, A. K.; Bordenyuk, A. N.; Benderskii, A. V. Effect of Nanoscale Geometry on Molecular Conformation: Vibrational Sum-Frequency Generation of Alkanethiols on Gold Nanoparticles. J. Am. Chem. Soc. 2006, 128, 14244-14245.

(14) Bordenyuk, A. N.; Weeraman, C.; Yatawara, A.; Jayathilake, H. D.; Stiopkin, I.; Liu, Y.; Benderskii, A. V. Vibrational Sum Frequency Generation Spectroscopy of Dodecanethiol on Metal Nanoparticles. J. Phys. Chem. C 2007, 111, 8925-8933.

(15) Kawai, T.; Neivandt, D. J.; Davies, P. B. Sum Frequency Generation on Surfactant-Coated Gold Nanoparticles. J. Am. Chem. Soc. 2000, 122, 12031-12032.

(16) Tourillon, G.; Dreesen, L.; Volcke, C.; Sartenaer, Y.; Thiry, P. A.; Peremans, A. Total Internal Reflection Sum-Frequency Generation Spectroscopy and Dense Gold Nanoparticles Monolayer: A Route for Probing Adsorbed Molecules. Nanotechnology 2007, 18, 415301.

(17) Li, J.-F.; Zhang, Y.-J.; Ding, S.-Y.; Panneerselvam, R.; Tian, Z.-Q. Core-Shell Nanoparticle-Enhanced Raman Spectroscopy. Chem. Rev. 2017, 117, 5002-5069.

(18) Ghalgaoui, A.; Ouvrard, A.; Wang, J.; Carrez, S.; Zheng, W.; Bourguignon, B. Electron to Adsorbate Energy Transfer in Nanoparticles: Adsorption Site, Size, and Support Matter. J. Phys. Chem. Lett. 2017, 8, 2666-2671.

(19) Ouvrard, A.; Ghalgaoui, A.; Michel, C.; Barth, C.; Wang, J.; Carrez, S.; Zheng, W.; Henry, C. R.; Bourguignon, B. CO Chemisorption on Ultrathin MgO-Supported Palladium Nanoparticles. J. Phys. Chem. C 2017, 121, 5551-5564.

(20) Busson, B.; Tadjeddine, A. Non-Uniqueness of Parameters Extracted from Resonant Second-Order Nonlinear Optical Spectroscopies. J. Phys. Chem. C 2009, 113, 2189521902.

(21) Sovago, M.; Vartiainen, E.; Bonn, M. Determining Absolute Molecular Orientation at Interfaces: A Phase Retrieval Approach for Sum Frequency Generation Spectroscopy. $J$. Phys. Chem. C 2009, 113, 6100-6106.

(22) Meier, M.; Wokaun, A. Enhanced Fields on Large Metal Particles: Dynamic Depolarization. Opt. Lett. 1983, 8, 581-583.

(23) de Beer, A. G. F.; Roke, S. Nonlinear Mie Theory for Second-Harmonic and SumFrequency Scattering. Phys. Rev. B 2009, 79, 155420.

(24) Roy, S.; Hung, K.-K.; Stege, U.; Hore, D. K. Rotations, Projections, Direction Cosines, and Vibrational Spectra. Appl. Spectrosc. Rev. 2014, 49, 233-248.

(25) Andrews, S. S. Using Rotational Averaging To Calculate the Bulk Response of Isotropic and Anisotropic Samples from Molecular Parameters. J. Chem. Educ. 2004, 81, 877-885.

(26) Zhuang, X.; Miranda, P. B.; Kim, D.; Shen, Y. R. Mapping Molecular Orientation and 
Conformation at Interfaces by Surface Nonlinear Optics. Phys. Rev. B 1999, 59, 1263212640 .

(27) Watanabe, N.; Yamamoto, H.; Wada, A.; Domen, K.; Hirose, C.; Ohtake, T.; Mino, N. Vibrational Sum-Frequency Generation (VSFG) Spectra of N-Alkyltrichlorosilanes Chemisorbed on Quartz Plate. Spectrochim. Acta Part A-Molecular Biomol. Spectrosc. 1994, 50, 1529-1537.

(28) Jena, K. C.; Hung, K. K.; Schwantje, T. R.; Hore, D. K. Methyl Groups at Dielectric and Metal Surfaces Studied by Sum-Frequency Generation in Co- and Counter-Propagating Configurations. J. Chem. Phys. 2011, 135, 044704.

(29) Lu, R.; Gan, W.; Wu, B. H.; Zhang, Z.; Guo, Y.; Wang, H. F. C-H Stretching Vibrations of Methyl, Methylene and Methine Groups at the Vapor/Alcohol $(\mathrm{N}=1-8)$ Interfaces. J. Phys. Chem. B 2005, 109, 14118-14129.

(30) Dalstein, L.; Revel, A.; Humbert, C.; Busson, B. Nonlinear Optical Response of a Gold Surface in the Visible Range: A Study by Two-Color Sum-Frequency Generation Spectroscopy. I. Experimental Determination. J. Chem. Phys. 2018, 148, 134701.

(31) Chen, X.; Wang, J.; Boughton, A. P.; Kristalyn, C. B.; Chen, Z. Multiple Orientation of Melittin inside a Single Lipid Bilayer Determined by Combined Vibrational Spectroscopic Studies. J. Am. Chem. Soc. 2007, 129, 1420-1427.

(32) Yeganeh, M. S.; Dougal, S. M.; Silbernagel, B. G. Sum Frequency Generation Studies of Surfaces of High-Surface-Area Powdered Materials. Langmuir 2006, 22, 637-641.

(33) Belkin, M. A.; Kulakov, T. A.; Ernst, K. H.; Yan, L.; Shen, Y. R. Sum-Frequency Vibrational Spectroscopy on Chiral Liquids: A Novel Technique to Probe Molecular Chirality. Phys. Rev. Lett. 2000, 85, 4474-4477.

(34) Steel, A. B.; Herne, T. M.; Tarlov, M. J. Electrochemical Quantitation of DNA Immobilized on Gold. Anal. Chem. 1998, 70, 4670-4677.

(35) Tao, Y.-T.; Wu, C.-C.; Eu, J.-Y.; Lin, W.-L.; Wu, K.-C.; Chen, C. Structure Evolution of Aromatic-Derivatized Thiol Monolayers on Evaporated Gold. Langmuir 1997, 13, 40184023.

(36) Levicky, R.; Herne, T. M.; Tarlov, M. J.; Satija, S. K. Using Self-Assembly To Control the Structure of DNA Monolayers on Gold: A Neutron Reflectivity Study. J. Am. Chem. Soc. 1998, 120, 9787-9792.

(37) Poirier, G. E. Characterization of Organosulfur Molecular Monolayers on Au(111) Using Scanning Tunneling Microscopy. Chem. Rev. 1997, 97, 1117-1128.

(38) Benghorieb, S.; Saoudi, R.; Tishchenko, A. V. Extraction of the 3D Plasmon Field. Plasmonics 2011, 6, 445-455.

(39) Iida, K.; Noda, M.; Nobusada, K. Interface Electronic Properties Between a Gold Core and Thiolate Ligands: Effects on an Optical Absorption Spectrum in Au 133 (SPh-TBu) 52. J. Phys. Chem. C 2016, 120, 2753-2759.

(40) Creighton, J. A. Surface Raman Electromagnetic Enhancement Factors for Molecules at 
the Surface of Small Isolated Metal Spheres: The Determination of Adsorbate Orientation from Sers Relative Intensities. Surf. Sci. 1983, 124, 209-219.

(41) Bobbert, P. A.; Vlieger, J. Physica A: Theoretical and Statistical Physics. Physica A 1987, 147, 115-141.

(42) Román-Velázquez, C. E.; Noguez, C.; Barrera, R. G. Substrate Effects on the Optical Properties of Spheroidal Nanoparticles. Phys. Rev. B 2000, 61, 10427-10436.

(43) Okamoto, T.; Yamaguchi, I. Optical Absorption Study of the Surface Plasmon Resonance in Gold Nanoparticles Immobilized onto a Gold Substrate by Self-Assembly Technique. J. Phys. Chem. B 2003, 107, 10321-10324.

(44) Aravind, P. K.; Metiu, H. The Effects of the Interaction between Resonances in the Electromagnetic Response of a Sphere-Plane Structure; Applications to Surface Enhanced Spectroscopy. Surf. Sci. 1983, 124, 506-528.

(45) Aravind, P. K.; Metiu, H. Use of a Perfectly Conducting Sphere to Excite the Plasmon of a Flat Surface. 1. Calculation of the Local Field with Applications to Surface-Enhanced Spectroscopy. J. Phys. Chem. 1982, 86, 5076-5084.

(46) Noguez, C. Surface Plasmons on Metal Nanoparticles: The Influence of Shape and Physical Environment. J. Phys. Chem. C 2007, 111, 3806-3819.

(47) Truong, V. V.; Scott, G. D. Optical Properties of Aggregated Noble Metal Films. J. Opt. Soc. Am. 1977, 67, 502-510.

(48) Wind, M. M.; Vlieger, J.; Bedeaux, D. The Polarizability of a Truncated Sphere on a Substrate I. Physica A 1987, 141, 33-57.

(49) Freeman, R. G.; Grabar, K. C.; Allison, K. J.; Bright, R. M.; Davis, J. A.; Guthrie, A. P.; Hommer, M. B.; Jackson, M. A.; Smith, P. C.; Walter, D. G.; et al. Self-Assembled Metal Colloid Monolayers: An Approach to SERS Substrates. Science 1995, 267, 1629-1632.

(50) Hu, J.; Tanabe, M.; Sato, J.; Uosaki, K.; Ikeda, K. Effects of Atomic Geometry and Electronic Structure of Platinum Surfaces on Molecular Adsorbates Studied by Gap-Mode SERS. J. Am. Chem. Soc. 2014, 136, 10299-10307.

(51) Okuno, M.; Tokimoto, T.; Eguchi, M.; Kano, H.; Ishibashi, T. Intensity Enhancement of Vibrational Sum Frequency Generation by Gap-Mode Plasmon Resonance. Chem. Phys. Lett. 2015, 639, 83-87.

(52) Ikeda, K.; Suzuki, S.; Uosaki, K. Crystal Face Dependent Chemical Effects in SurfaceEnhanced Raman Scattering at Atomically Defined Gold Facets. Nano Lett. 2011, 11, 1716-1722.

(53) Edwards, D. F. Silicon. In Handbook of optical constants of solids; Palik, E. D., Ed.; Academic Press, 1985; pp 547-569.

(54) Ohno, P. E.; Wang, H.; Geiger, F. M. Second-Order Spectral Lineshapes from Charged Interfaces. Nat. Commun. 2017, 8, 1032.

(55) Gonella, G.; Lütgebaucks, C.; de Beer, A. G. F.; Roke, S. Second Harmonic and Sum- 
Frequency Generation from Aqueous Interfaces Is Modulated by Interference. J. Phys. Chem. C 2016, 120, 9165-9173.

(56) Wen, Y.-C.; Zha, S.; Liu, X.; Yang, S.; Guo, P.; Shi, G.; Fang, H.; Shen, Y. R.; Tian, C. Unveiling Microscopic Structures of Charged Water Interfaces by Surface-Specific Vibrational Spectroscopy. Phys. Rev. Lett. 2016, 116, 016101.

(57) Olmon, R. L.; Slovick, B.; Johnson, T. W.; Shelton, D.; Oh, S. H.; Boreman, G. D.; Raschke, M. B. Optical Dielectric Function of Gold. Phys. Rev. B 2012, 86, 235147.

(58) Yang, H. H. U.; D’Archangel, J.; Sundheimer, M. L.; Tucker, E.; Boreman, G. D.; Raschke, M. B. Optical Dielectric Function of Silver. Phys. Rev. B 2015, 91, 235137.

(59) Hövel, H.; Fritz, S.; Hilger, A.; Kreibig, U.; Vollmer, M. Width of Cluster Plasmon Resonances: Bulk Dielectric Functions and Chemical Interface Damping. Phys. Rev. B 1993, 48, 18178-18188.

(60) CRC Handbook of Chemistry and Physics, 97th editi.; Haynes, W. M., Ed.; CRC PRESS, 2016.

(61) Boujday, S.; Parikh, A. N.; Liedberg, B.; Song, H. Functionalization of Gold Nanoparticles. In Gold Nanoparticles for Physics, Chemistry and Biology; Louis, C., Pluchery, O., Eds.; World Scientific: London, 2017; pp 201-227.

(62) Badia, A.; Cuccia, L.; Demers, L.; Morin, F.; Lennox, R. B. Structure and Dynamics in Alkanethiolate Monolayers Self-Assembled on Gold Nanoparticles: A DSC, FT-IR, and Deuterium NMR Study. J. Am. Chem. Soc. 1997, 119, 2682-2692.

(63) Hostetler, M. J.; Stokes, J. J.; Murray, R. W. Infrared Spectroscopy of Three-Dimensional Self-Assembled Monolayers: N-Alkanethiolate Monolayers on Gold Cluster Compounds. Langmuir 1996, 12, 3604-3612.

(64) Guo, Z.; Zheng, W.; Hamoudi, H.; Dablemont, C.; Esaulov, V. A.; Bourguignon, B. On the Chain Length Dependence of CH3 Vibrational Mode Relative Intensities in Sum Frequency Generation Spectra of Self Assembled Alkanethiols. Surf. Sci. 2008, 602, $3551-3559$.

(65) Zhu, M.; Lerum, M. Z.; Chen, W. How To Prepare Reproducible, Homogeneous, and Hydrolytically Stable Aminosilane-Derived Layers on Silica. Langmuir 2012, 28, 416 423.

(66) Bossard-Giannesini, L.; Cruguel, H.; Lacaze, E.; Pluchery, O. Plasmonic Properties of Gold Nanoparticles on Silicon Substrates: Understanding Fano-like Spectra Observed in Reflection. Appl. Phys. Lett. 2016, 109, 111901.

(67) Raab, R. E.; de Lange, O. L. Multipole Theory in Electromagnetism; Oxford University Press: Oxford, 2005. 
TOC graphic

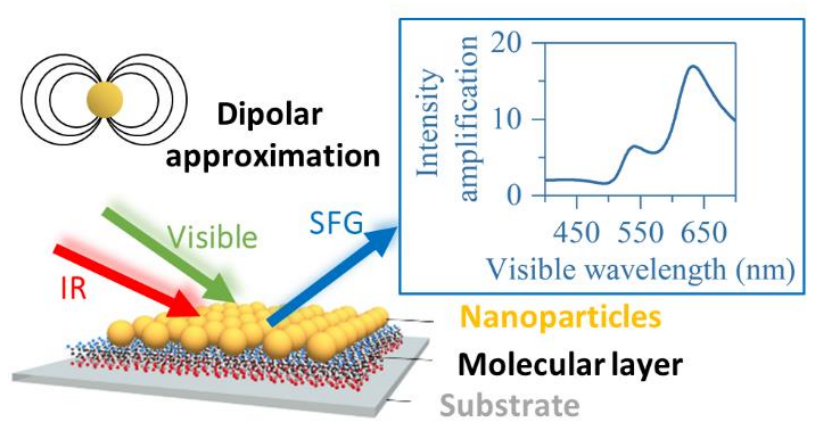

